\title{
Global transcriptional profiling of CD15+ granulocytes from patients with septic shock and systemic inflammatory response syndrome (SIRS).
}

\author{
Shahan Mamoor ${ }^{1}$, MS \\ 1Thomas Jefferson School of Law \\ San Diego, CA 92101 \\ correspondence: mamoorsk@tjsl.edu
}

Sepsis, the body's reaction to infection in what is normally a sterile bloodstream, is a major cause of mortality in the United States (1). I used a microarray data set from a cohort of thirtyone patients with septic shock or systemic inflammatory response syndrome (2) to determine the major transcriptional changes associated with each disease state. I found that globally, the granulocytes of patients with SIRS resembled that of patients with septic shock at the level of transcription. For many genes expressed in the granulocyte, SIRS represented an "intermediate" gene expression state between that of control patients and those of patients with septic shock. The identification of the most differentially expressed genes in the granulocytic immune cells of patients with septic shock can facilitate the development of novel therapeutics or diagnostics for a condition that, despite decades of research, possesses a $14.7 \%$ to $29.9 \%$ in-hospital mortality rate (1).

Keywords: SIRS, systemic inflammatory response syndrome, sepsis, septic shock, granulocyte, systems analysis of sepsis 


\section{Introduction}

Septic shock is a major cause of morbidity and mortality in the United States (1) and is characterized by an immune response to pathogens in the otherwise-sterile bloodstream (5). The time frame by which patients with septic shock die $(3,4)$ suggests that innate immune cells rather than adaptive immune cells (e.g., lymphocytes) drive this lethal pathology.

To understand that transcriptional framework that innate immune cells operate under in septic shock and a related, less-lethal pathology termed systemic inflammatory response syndrome, or SIRS (6), I utilized a public dataset generated by microarray analysis of granulocytic CD15+ innate immune cells to compare the global gene expression profiles of granulocytes from patients with septic shock, granulocytes from patients with SIRS, and granulocytes from control patients (granulocytes isolated from patients prior to surgery).

Interestingly, for a majority of the most differentially expressed genes in the septic granulocyte transcriptomes, the expression level of these genes in patients with SIRS was between that of the expression level in patients with septic shock and control patients. These findings suggest that, at least in granulocytes, SIRS represents an intermediate transcriptional state between homeostatic and septic.

\section{Methods}

Dataset GSE123731 (2) was used in conjunction with GEO2R to identify the two hundred and fifty most differentially expressed genes in the granulocytes (CD15+ cells) of patients with septic shock ( $n=15)$, systemic inflammatory response syndrome (SIRS) $(n=16)$, or from control granulocytes obtained from patients pre-surgery $(n=11)$. The RNA expression value of each of these genes from each patient in each cohort was extracted. P-values were adjusted using the "Benjamini and Hochberg" method, and log-transformation of data was set 
to "Auto-detect". Statistical analysis comparing the mean RNA expression values of each group to one another was conducted by one-way ANOVA analysis (PRISM 8).

\section{Results}

To understand the major transcriptional changes that occur in the immune cells of patients with septic shock and patients with SIRS, I utilized a publicly available dataset generated by microarray analysis of isolated CD15+ granulocytes from pre-surgical patients (control), CD15+ granulocytes from patients with SIRS, and CD15+ granulocytes from patients with septic shock. Of the 250 most differentially expressed genes in the sepsis granulocyte transcriptome, 199 could be classified as possessing "SIRS Intermediate" expression, or having a level of expression in SIRS patients that was graded between the expression level of that gene in granulocytes from patients with septic shock and granulocytes of control patients, and significantly so (Figure 20). If the expression level of that gene was higher in the granulocytes of patients with septic shock than in the granulocytes of control patients, the expression level of that gene in granulocytes of SIRS patients was higher than in the granulocytes of control patients but lower than in the granulocytes of patients with septic shock. Conversely, if the expression level of that gene was lower in the granulocytes of patients with septic shock than in the granulocytes of control patients, the expression level of that gene in granulocytes of SIRS patients was lower than in the granulocytes of control patients but higher than in the granulocytes of patients with septic shock.

Surprisingly, three members of the MEF family of transcription factor family were among the most differentially expressed genes in the granulocytes of patients with SIRS and septic shock when compared to the granulocytes of control patients. 
The expression of both MEF2C and MEF2D were significantly different between control and patients with systemic inflammatory response syndrome (SIRS), and granulocytes from patients with SIRS or septic shock.

The expression of MEF2C and MEF2D was significantly higher in granulocytes from control patients (Figure 1; $\mathrm{p}<0.0001$ ), and significantly higher in the granulocytes of patients with SIRS versus patients with septic shock (Figure 1; $p<0.0001$ ). The expression of MEF2A was significantly lower in the granulocytes of control patients when compared to the granulocytes of patients with SIRS or patients with septic shock (Figure $1 ; p<0.0001)$ ), but did not significantly differ between patients with SIRS and patients with septic shock (Figure 1; $p=0.3229)$.

Two other transcription factors were identified as among the most differentially expressed genes in the granulocytes of patients with SIRS and septic shock when compared to the granulocytes of control patients.

TCF20 and RUNX1 displayed opposing patterns of differential expression in the granulocytes of patients with SIRS and septic shock. TCF20 was expressed at significantly lower levels in the granulocytes of patients with septic shock than in both the granulocytes of patients with SIRS and in the granulocytes of control patients (Figure 2; $p<0.0001$ and $p<0.0001$, respectively), with significantly lower expression of TCF20 in the granulocytes of SIRS patients when compared to the granulocytes of control patients (Figure 2; p,0.0001). RUNX1 was expressed at significantly higher levels in the granulocytes of patients with septic shock than in both the granulocytes of patients with SIRS and the granulocytes of control patients (Figure 2; $p<0.0001$ and $p<0.0001$, respectively), with significantly higher expression of RUNX1 in the granulocytes of SIRS patients when compared to the granulocytes of control patients (Figure 2; $\mathrm{p}=0.0006$ ). 
Together, these data demonstrate that transcription factors are among the most differentially expressed genes in the granulocytes of patients with septic shock and the granulocytes of patients with SIRS when compared to the granulocytes of control patients.

Transcriptional regulators and epigenetic modifiers were found to be differentially expressed in the transcriptomes of granulocytes from patients with septic shock and SIRS relative to the granulocytes of control patients.

ARID5B, GADD45A and METTL9 were expressed at significantly higher levels in the granulocytes of patients with septic shock when compared to the granulocytes of patients with SIRS or septic shock, with significantly higher expression of ARID5B, GADD45A and METTL9 in the granulocytes of patients with septic shock when compared to the granulocytes of patients with SIRS (Figure 3; $p<0.0001$ for all comparisons except for GADD45: SIRS vs. septic shock, $p=0.0321$, and METTL9: SIRS vs. septic shock, $p=0.0010)$. CEBPA was also expressed at significantly higher levels in the granulocytes of patients with septic shock or SIRS when compared to the granulocytes of control patients (Figure 3; $p<0.0001$ and $p<0.0001$, respectively), but the expression of CEBPA was not significantly different between patients with SIRS and septic shock (Figure 3; $p=0.0861$ ).

The expression of two other epigenetic molecules, YTHDC1 and HIST2HBC were significantly lower in the granulocytes of patients with septic shock and in the granulocytes of patients with SIRS when compared to the granulocytes of control patients (Figure 3; $p<0.0001$ for all comparisons). The expression of YTHDC1 and HIST2HBC were also significantly lower in the granulocytes of patients with septic shock when compared to the granulocytes of patients with SIRS (Figure 3; $p<0.0001$ for both comparisons). 
Overall, these data demonstrate that epigenetic molecules and transcriptional regulators are among the most differentially expressed genes in the granulocytes of patients with septic shock and the granulocytes of patients with SIRS when compared to the granulocytes of control patients.

Genes that encode molecules responsible for the nuclear transport of cellular cargoes were also among those most differentially expressed between the granulocytes of control when compared to the granulocytes of patients with SIRS or septic shock. One alpha karyopherin, KPNA6, one beta karyopherin, TNPO3, and a Ran-binding protein, RANBP9, were all expressed at significantly higher levels in the granulocyte transcriptome of patients with SIRS and septic shock relative to the granulocytes of control patients (Figure 4; $p<0.0001$ for all comparisons). While the expression of KPNA6 and RANBP9 was significantly higher in patients with septic shock than in patients with SIRS (Figure 4; $\mathrm{p}<0.0001$ and $\mathrm{P}=0.0195$, respectively), the expression of TNPO3 was not significantly different when comparing the granulocytes of patients with SIRS and patients with septic shock (Figure 4; $p=0.0714$ ).

Transcripts for proteins involved in vesicular trafficking were among the most differentially expressed when comparing the granulocyte transcriptome in patients with SIRS and septic shock relative to the granulocytes of control patients.

The expression of SEC14L1 and DOCK5 was significantly higher in granulocytes from control patients when compared to the granulocytes of patients with SIRS or patients with septic shock (Figure 5; $p<0.0001$ and $p<0.0001$, respectively), and significantly higher in the granulocytes of patients with SIRS versus patients with septic shock (Figure $5 ; p<0.0001$ ). 
Contrarily, expression of SNX3, VPS54, SEC24A, and RAB27A was significantly lower in the granulocytes of control patients when compared to the granulocytes of patients with SIRS and patients with septic shock, respectively (Figure 5; SNX3: $p<0.0001$ and $p<0.0001$; VPS54: $p<0.0001$ and $p<0.0001$; SEC24A: $p<0.0001$ and $p<0.0001$; and RAB27A: $p<0.0001$ and $\mathrm{p}<0.0001$, respectively). The expression of SNX3, VPS54, SEC24A, and RAB27A was significantly higher in the granulocytes of patients with septic shock when compared to the granulocytes of patients with SIRS (Figure 5; SNX3: $p=0.0008$; VPS54: $p<0.0001$; SEC24A: $p<0.0001$; and RAB27A: $p<0.0001)$.

Overall, these data demonstrate that genes encoding molecules responsible for the cellular transport of molecules are among the most differentially expressed in the granulocytes of patients with septic shock and the granulocytes of patients with SIRS when compared to the granulocytes of control patients.

Cluster of differentiation (CD) genes are useful as diagnostic markers as their expression on the cell surface permits the use of antibodies and antibody-like reagents for diagnostic purposes. CD genes were also identified as among the genes most differentially expressed in the granulocytes of patients with SIRS and septic shock relative to the granulocytes of control patients.

CD44, CD63, and CD177 were all expressed at significantly higher levels in the granulocytes of patients with septic shock when compared to the granulocytes of control patients, and significantly higher in the granulocytes of patients with septic shock when compared to the granulocytes of patients with SIRS (Figure 6; $p<0.0001$ for all comparisons except for CD44: septic shock vs. SIRS, $p=0.0109$, and CD177: septic shock vs SIRS, $\mathrm{p}=0.0031$ ). Contrarily, CD300LB was expressed at significantly lower levels in the granulocytes of patients with septic shock and patients with SIRS when compared to the granulocytes of 
control patients (Figure 6; $p<0.0001$ for all comparisons). CD300LB was also expressed at lower levels in the granulocytes of patients with septic shock when compared to the granulocytes of patients with SIRS (Figure 6; $p=0.0176$ ).

Molecules involved in signal transduction were among most differentially expressed genes in the granulocytes of patients with SIRS and septic shock when compared to the granulocytes of control patients.

The NOTCH1 signaling molecule was expressed at significantly lower levels in the granulocytes of patients with septic shock and the granulocytes of patients with SIRS when compared to the granulocytes of control patients (Figure 7; $p<0.0001$ and $p=0.0006$, respectively). NOTCH1 was also expressed at significantly lower levels in the granulocytes of patients with septic shock when compared to the granulocytes of patients with SIRS (Figure 7; $\mathrm{p}<0.0001)$

Two MAP kinase signaling effectors, MAPK14 and MAP2K6 were among the most differentially expressed genes in the granulocytes of patients with SIRS and septic shock relative to the granulocytes of control patients. MAPK14 and MAP2K6 were both expressed at significantly higher levels in the granulocytes of patients with septic shock and the granulocytes of patients with SIRS when compared to the granulocytes of control patients (Figure 8; $\mathrm{p}<0.0001$ for all comparisons). MAPK14 and MAP2K6 were also expressed at significantly higher levels in the granulocytes of patients with septic shock when compared to the granulocytes of patients with SIRS (Figure 8; $p=0.0022$ and $p=0.0162$, respectively).

Two matrix metalloproteinases were among the genes whose expression was most differentially regulated in the granulocytes of patients with SIRS and septic shock when compared to the granulocytes of control patients. 
MMP8 and MMP9 were both expressed at significantly higher levels in the granulocytes of patients with septic shock and the granulocytes of patients with SIRS when compared to the granulocytes of control patients (Figure 9; $p<0.0001$ for all comparisons). MMP8 and MMP9 were also expressed significantly higher in the granulocytes of patients with septic shock when compared to the granulocytes of patients with SIRS (Figure 9; $p<0.0001$ and $p=0.0054$, respectively).

Protein kinase AKT1 and RICTOR, a component of the kinase that phosphorylates AKT were among the genes most differently expressed in the granulocytes of patients with SIRS and septic shock.

AKT1 and RICTOR were both expressed at significantly lower levels in the granulocytes of patients with septic shock and the granulocytes of patients with SIRS when compared to the granulocytes of control patients (Figure 10; $p<0.0001$ for all comparisons). AKT1 and RICTOR were also expressed at significantly lower levels in the granulocytes of patients with septic shock when compared to the granulocytes of patients with SIRS (Figure 10; $p<0.0001$ ).

Three ubiquitin-related transcripts were identified among the most differentially expressed in the granulocytes of patients with SIRS and septic shock when compared to the granulocytes of control patients.

USP47 and USP4 displayed opposing patterns of differential expression, with higher expression of USP47 in the granulocytes of patients with septic shock and SIRS when compared with the granulocytes of control patients (Figure 11; $\mathrm{p}<0.0001$ for both comparisons), but lower expression of USP4 in the granulocytes of patients with septic shock and the granulocytes of patients with SIRS when compared to the granulocytes of control 
patients (Figure 11; $p<0.0001$ for both comparisons). USP47 was expressed at significantly higher levels in the granulocytes of patients with septic shock when compared to the granulocytes of patients with SIRS (Figure 11; $<<0.0001$ ), while USP4 was expressed at significantly lower levels in the granulocytes of patients with septic shock when compared to the granulocytes of patients with SIRS (Figure 11; $p<0.0001$ ).

Genes encoding receptors that recognize ligands for transduction of signals across the plasma membrane were also identified as being differentially expressed by the granulocytes of patients with septic shock and SIRS.

Activin receptor ACVR1B and the colony signaling factor receptor CSF1R were among the genes whose expression was most differentially regulated when comparing the granulocytes of patients with SIRS and septic shock relative to the granulocytes of control patients. ACVR1B was expressed at significantly higher levels in the granulocytes of patients with septic shock and the granulocytes of patients with SIRS when compared to the granulocytes of control patients (Figure 12; $\mathrm{p}<0.0001$ for all comparisons), while CSF1R was expressed at significantly lower levels in the granulocytes of patients with septic shock and the granulocytes of patients with SIRS when compared to the granulocytes of control patients (Figure 12; $p<0.0001$ for all comparisons). ACVR1B was expressed at significantly higher levels in the granulocytes of patients with septic shock when compared to the granulocytes of patients with SIRS (Figure 12; $p<0.0232$ ) while CSF1R was expressed at significantly lower levels in the granulocytes of patients with septic shock when compared to the granulocytes of patients with SIRS (Figure 12; $p<0.0001)$.

The Fc fragment of IgA receptor, FCAR and the Fc fragment of the high-affinity lgE receptor, FCER1G were also among the genes whose expression was most differentially 
expressed in patients with SIRS and septic shock when compared to the granulocytes of control patients.

FCAR and FCER1G were both expressed at significantly higher levels in the granulocytes of patients with SIRS and the granulocytes of patients with septic shock when compared to the granulocytes of control patients (Figure 13; $\mathrm{p}<0.0001$ for all comparisons). While FCER1G was expressed at significantly higher levels in the granulocytes of patients with septic shock when compared to the granulocytes of patients with SIRS (Figure 13; $p=0.0050$ ), FCAR was not (Figure 13; $\mathrm{p}=0.0563$ ).

Four innate immune cell surface receptors was differentially expressed in the transcriptomes of granulocytes from patients with SIRS and septic shock when compared to the granulocytes of control patients

CR1, TLR5, and CLEC4D were all expressed at significantly higher levels in the granulocytes of patients with septic shock and the granulocytes of patients SIRS when compared to the granulocytes of control patients (Figure 14; $p<0.0001$ for all comparisons). While CLEC4D was expressed at significantly higher levels in the granulocytes of patients with septic shock when compared to the granulocytes of patients with SIRS (Figure 14; $p=0.0053$ ), CR1 and TLR5 were not (Figure 14; $\mathrm{p}=0.1604$ and $\mathrm{p}=0.9682$, respectively). Contrarily, CLEC7A was expressed at significantly lower levels in the granulocytes of patients with septic and SIRS when compared to the granulocytes of control patients (Figure 14; $p<0.0001$ for both comparisons). CLEC7A was also expressed at significantly lower levels in the granulocytes of patients with septic shock when compared to the granulocytes of patients with SIRS (Figure $14 ; p=0.0008)$. 
Four intracellular effectors of innate immune signaling were also differentially expressed by granulocytes in patients with SIRS and septic shock when compared to the granulocytes of control patients.

NLRC4, CARD6 and IRAK3 were all expressed at significantly higher levels in the granulocytes of patients with septic shock and the granulocytes of patients with SIRS when compared to the granulocytes of control patients (Figure 15; $\mathrm{p}<0.0001$ for all comparisons). The expression of NLRC4, CARD6 and IRAK3 were also significantly higher in the granulocytes of patients with septic shock when compared to the granulocytes of patients with SIRS (Figure 15; $p<0.0001, p=0.0050$, and $p=0.0110$, respectively).

IKBIP was expressed at significantly lower levels in the granulocytes of patients with septic shock and the granulocytes of patients with SIRS when compared to the granulocytes of control patients (Figure 15; $p=0.0001$ and $p=0.0025$, respectively). Expression of IKBIP was also significantly lower in the granulocytes of patients with septic shock when compared to the granulocytes of patients with SIRS (Figure 15; $p<0.0001$ ).

Strikingly, three fatty acid palmitoyltransferases and a mannosyltransferase were among the genes whose expression was most differentially regulated in granulocytes from patients with septic shock and SIRS when compared to the granulocytes of control patients.

Palmitoyltransferases ZDHHC2, ZDHHC3 and ZDHHC20 were all expressed at significantly higher levels in the granulocytes of patients with septic shock and the granulocytes of patients with SIRS when compared to the granulocytes of control patients (Figure 16; $p<0.0001$ for all comparisons). While the expression of ZDHHC3 and ZDHHC20 was significantly higher in the granulocytes of patients with septic shock when compared to 
the granulocytes of patients with SIRS (Figure 16; $p=0.0033$ and $p=0.0003$ ), the expression of ZDHHC2 was not (Figure 16; $p=0.2051)$.

Mannosyltransferase DPY19L3 was expressed at significantly higher levels in the granulocytes of patients with septic shock and the granulocytes of patients with SIRS when compared to the granulocytes of control patients (Figure 16; $\mathrm{p}<0.0001$ for both comparisons). Expression of DPY19L3 was not significantly different between granulocytes from patients with septic shock and the granulocytes of patients with SIRS (Figure 16; $p=0.2525$ ).

An ATP flippase and an ATPase were identified as differentially expressed in the granulocytes of patients with SIRS and septic shock relative to the granulocytes of control patients. ATP9A and ATP11B were both expressed at significantly higher levels in the granulocytes of patients with septic shock and the granulocytes of patients with SIRS when compared to the granulocytes of control patients (Figure 17; $\mathrm{p}<0.0001$ for all comparisons). ATP9A and ATP11B were both expressed at significantly higher levels in the granulocytes of patients with septic shock when compared to the granulocytes of patients with SIRS (Figure $17 ; p=0.0015$ and $p=0.0095$, respectively).

Together, these data suggest that enzymes involved in palmitoylation, mannosylation, and in ATP metabolism are among the genes most differentially expressed when comparing the granulocytes of patients with SIRS and the granulocytes of patients with septic shock to the granulocytes of control patients.

Two ribonucleoproteins were among the genes most differentially expressed in the granulocytes of patients with SIRS and septic shock when compared to the granulocytes of control patients. 
SNRNP70 and HNRNPH3 were both expressed at significantly lower levels in the granulocytes of patients with septic shock and the granulocytes of patients with SIRS when compared to the granulocytes of control patients (Figure 18; $p<0.0001$ for all comparisons except HNRNPH3 control vs. SIRS, $\mathrm{p}=0.0003)$. The expression of SNRNP70 and HNRNPH3 was significantly lower in the granulocytes of patients with septic shock when compared to the granulocytes of patients with SIRS (Figure 18; $p<0.0001$ for both comparisons)

Finally, two transcripts that are predicted to lack protein-coding potential were among the two hundred and fifty genes whose expression was most differentially regulated when comparing the transcriptomes of granulocytes from patients with SIRS and septic shock. PCBP-AS1 and PTOV-AS1 were both expressed at significantly lower levels in the granulocytes of patients with septic shock and the granulocytes of patients with SIRS when compared to the granulocytes of control patients (Figure 19; $\mathrm{p}<0.0001$ for all comparisons). PCBP-AS1 and PTOV-AS1 were also expressed at significantly lower levels in the granulocytes of patients with septic shock when compared to the granulocytes of patients with SIRS (Figure 19; $p<0.0001$ and $\mathrm{p}=0.0004$, respectively).

\section{Discussion}

I compared the global gene expression profiles of the granulocytic immune cells of patients with septic shock and patients with SIRS with that of the granulocytic immune cells of control patients using a public dataset generated by microarray analysis of sorted CD15+ cells from each patient population. From this analysis I identified the two hundred and fifty most differentially expressed genes in the granulocytes of patients with septic shock and in the granulocytes of patients with SIRS. These include transcription factors, epigenetic modifiers and transcriptional regulators, molecules involved in the transport of cargoes throughout the cell, cluster of differentiation markers genes encoding proteins responsible for signal 
transduction, innate immunity effectors, enzymes involved in post-translational modifications and ATP biology, ribonucleoproteins, non-coding RNA and others.

These analyses revealed that SIRS represents an intermediate transcriptional state in granulocytes as they progress from homestasis to sepsis. The identification of these differentially expressed genes will be useful to further understand the transcriptional landscape of immune cells in septic shock and for the discovery of therapeutic targets in a condition where $14-29 \%$ of patients will not leave the hospital alive.

\section{References}

1. Gaieski, D.F., Edwards, J.M., Kallan, M.J. and Carr, B.G., 2013. Benchmarking the incidence and mortality of severe sepsis in the United States. Critical care medicine, 41(5), pp.1167-1174.

2. Coulibaly, A., Velásquez, S.Y., Sticht, C., Figueiredo, A.S., Himmelhan, B.S., Schulte, J., Sturm, T., Centner, F.S., Schöttler, J.J., Thiel, M. and Lindner, H.A., 2019. AKIRIN1: A Potential New Reference Gene in Human Natural Killer Cells and Granulocytes in Sepsis. International journal of molecular sciences, 20(9), p.2290.

3. Mouncey, P.R., Osborn, T.M., Power, G.S., Harrison, D.A., Sadique, M.Z., Grieve, R.D., Jahan, R., Harvey, S.E., Bell, D., Bion, J.F. and Coats, T.J., 2015. Trial of early, goal-directed resuscitation for septic shock. New England Journal of Medicine, 372(14), pp.1301-1311.

4. Gaieski, D.F., Mikkelsen, M.E., Band, R.A., Pines, J.M., Massone, R., Furia, F.F., Shofer, F.S. and Goyal, M., 2010. Impact of time to antibiotics on survival in patients with severe sepsis or septic shock in whom early goal-directed therapy was initiated in the emergency department. Critical care medicine, 38(4), pp.1045-1053.

5. Cohen, J., 2002. The immunopathogenesis of sepsis. Nature, 420(6917), p.885.

6. Rangel-Frausto, M.S., Pittet, D., Costigan, M., Hwang, T., Davis, C.S. and Wenzel, R.P., 1995. The natural history of the systemic inflammatory response syndrome (SIRS): a prospective study. Jama, 273(2), pp.117-123. 

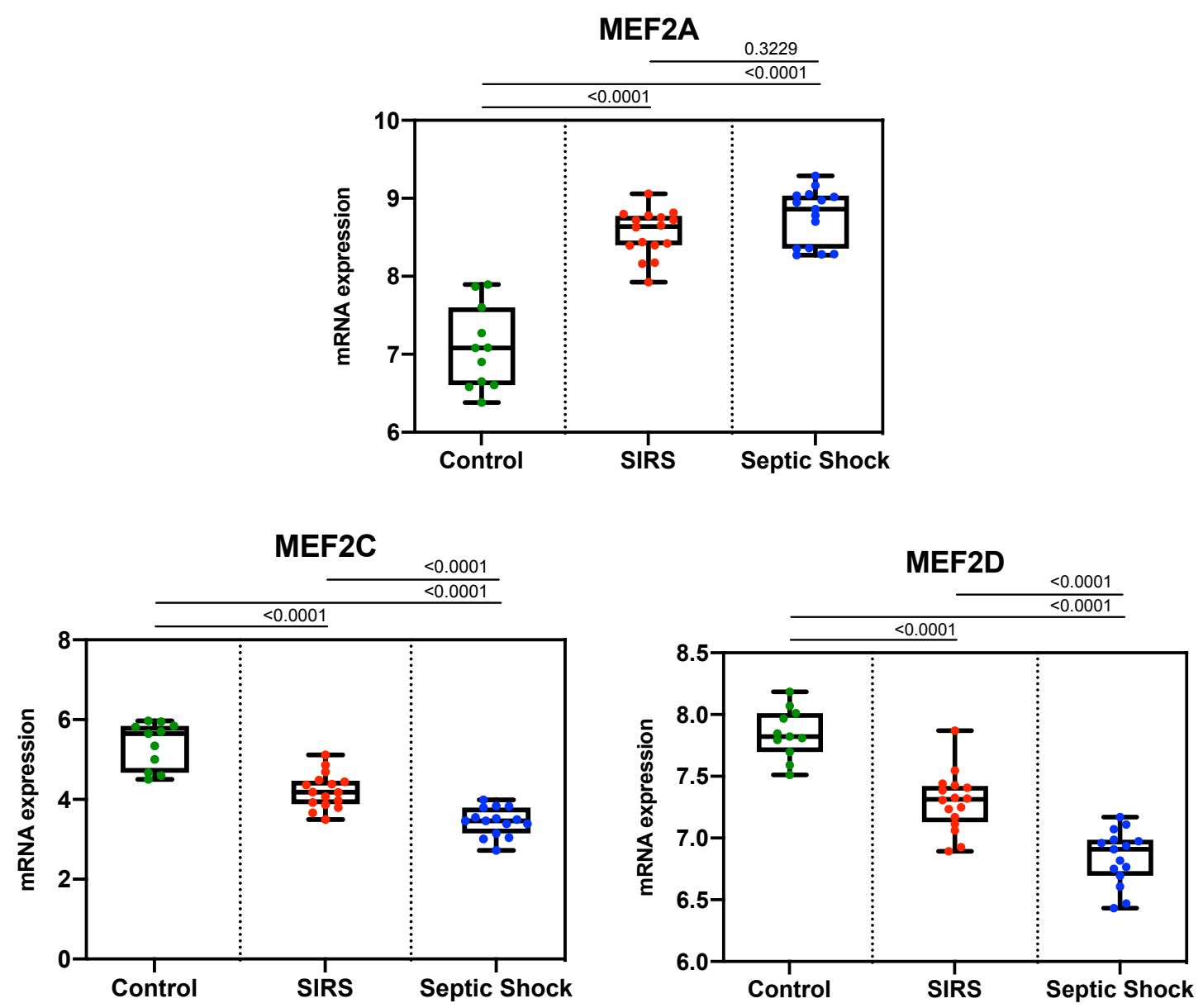

Figure 1: MEF2 family transcription factors are differentially expressed in the granulocytes of patients with SIRS and septic shock.

The expression of each gene in the granulocytes of control patients $(n=11)$, patients with SIRS $(n=16)$, or patients with septic shock $(n=15)$ is graphically depicted by box and whisker plot. One-way ANOVA analysis comparing the mean RNA expression value of each gene by patient group (control vs. SIRS, control vs. septic shock, and SIRS vs. septic shock) was performed and the exact $p$-value is indicated on each respective comparison. 

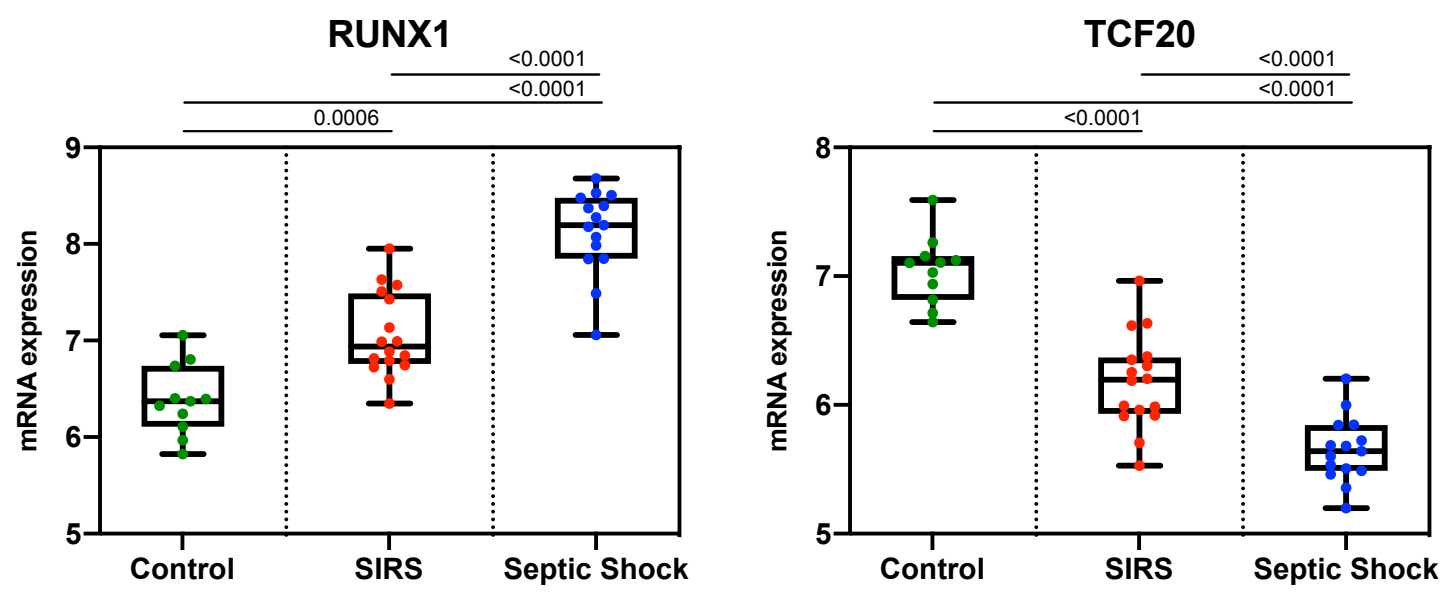

Figure 2: RUNX1 and TCF20 transcription factors are differentially expressed in the granulocytes of patients with SIRS and septic shock.

The expression of each gene in the granulocytes of control patients $(n=11)$, patients with SIRS $(n=16)$, or patients with septic shock $(n=15)$ is graphically depicted by box and whisker plot. One-way ANOVA analysis comparing the mean RNA expression value of each gene by patient group (control vs. SIRS, control vs. septic shock, and SIRS vs. septic shock) was performed and the exact $p$-value is indicated on each respective comparison. 

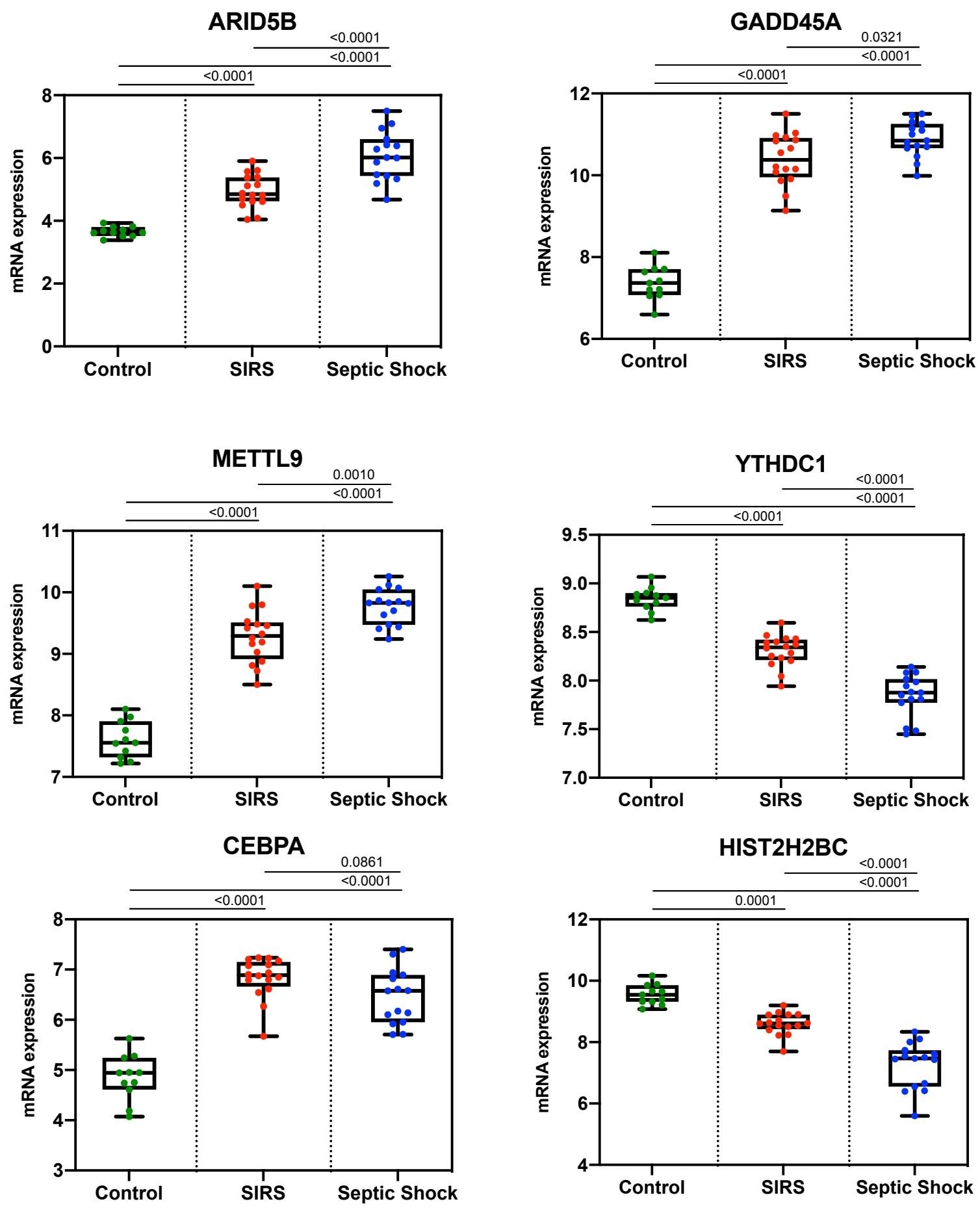

Figure 3: Transcriptional regulators and epigenetic molecules are differentially expressed in the granulocytes of patients with SIRS and septic shock.

The expression of each gene in the granulocytes of control patients $(n=11)$, patients with SIRS $(n=16)$, or patients with septic shock $(n=15)$ is graphically depicted by box and whisker plot. One-way ANOVA analysis comparing the mean RNA expression value of each gene by patient group (control vs. SIRS, control vs. septic shock, and SIRS vs. septic shock) was performed and the exact $\mathrm{p}$-value is indicated on each respective comparison. 

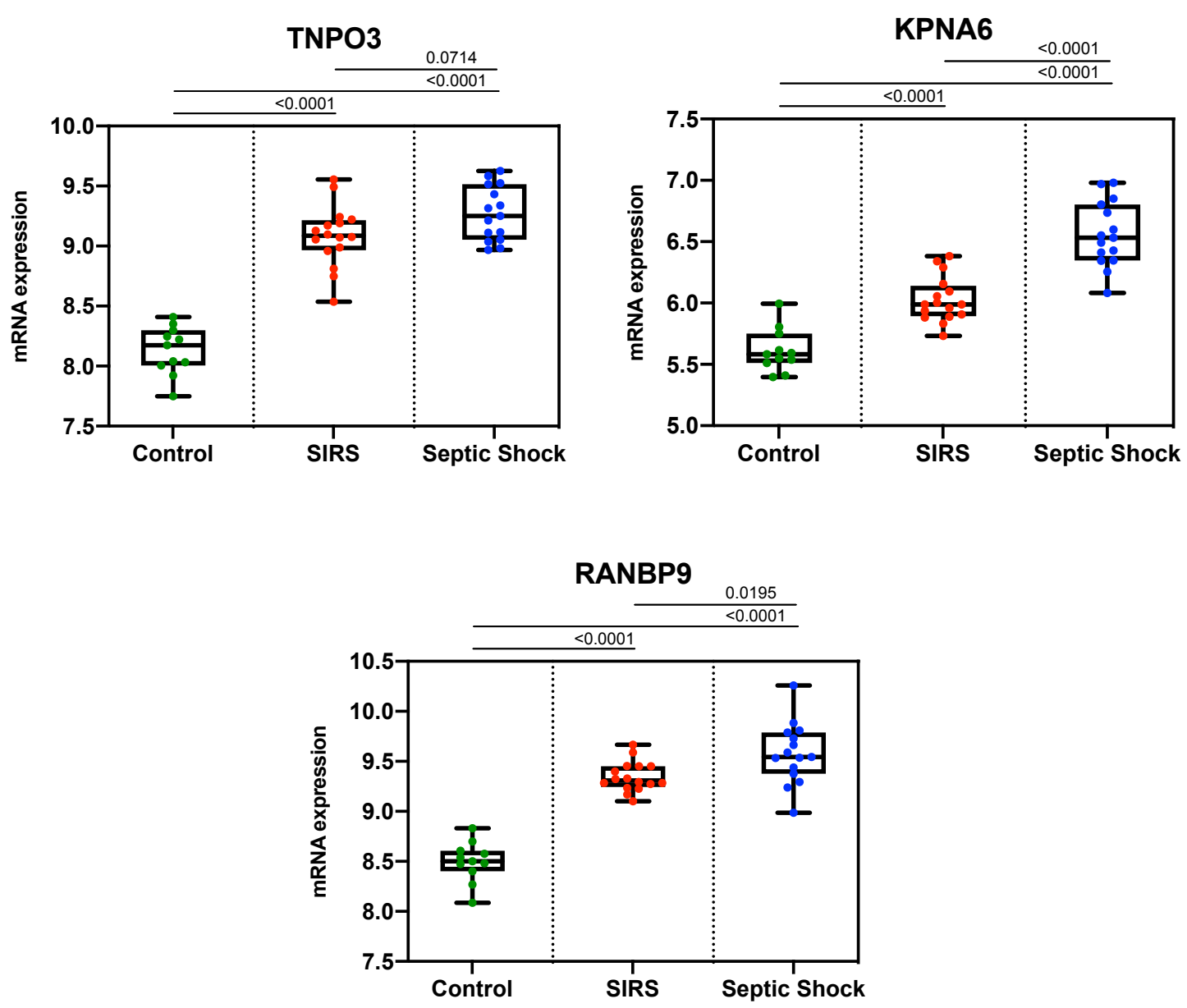

Figure 4: Genes encoding the transport of molecules into and out of the nucleus are differentially expressed in the granulocytes of patients with SIRS and septic shock.

The expression of each gene in the granulocytes of control patients $(n=11)$, patients with SIRS $(n=16)$, or patients with septic shock $(n=15)$ is graphically depicted by box and whisker plot. One-way ANOVA analysis comparing the mean RNA expression value of each gene by patient group (control vs. SIRS, control vs. septic shock, and SIRS vs. septic shock) was performed and the exact $p$-value is indicated on each respective comparison. 

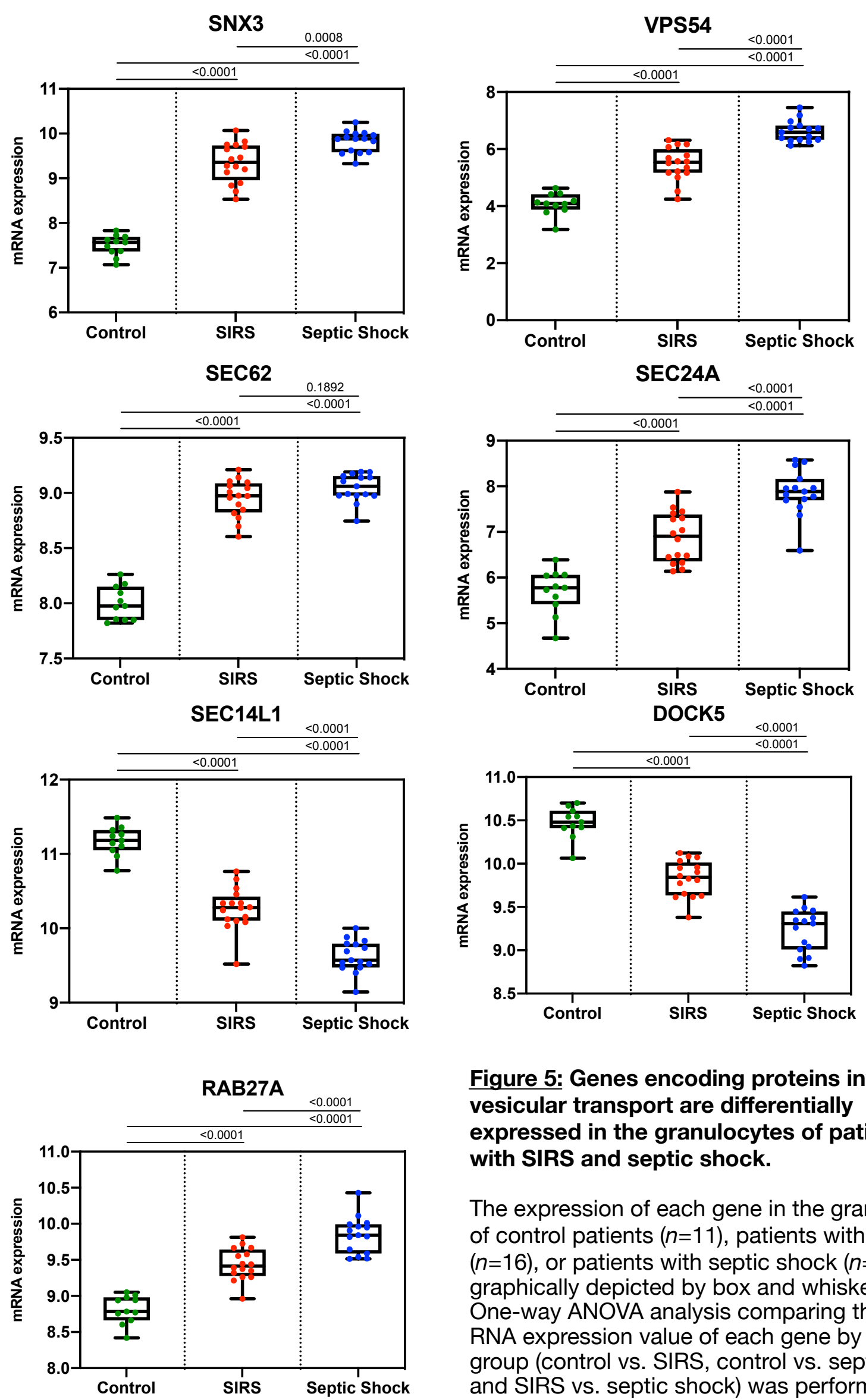

Figure 5: Genes encoding proteins involved in vesicular transport are differentially expressed in the granulocytes of patients with SIRS and septic shock.

The expression of each gene in the granulocytes of control patients $(n=11)$, patients with SIRS $(n=16)$, or patients with septic shock $(n=15)$ is graphically depicted by box and whisker plot. One-way ANOVA analysis comparing the mean RNA expression value of each gene by patient group (control vs. SIRS, control vs. septic shock, and SIRS vs. septic shock) was performed and the exact $p$-value is indicated on each respective comparison. 

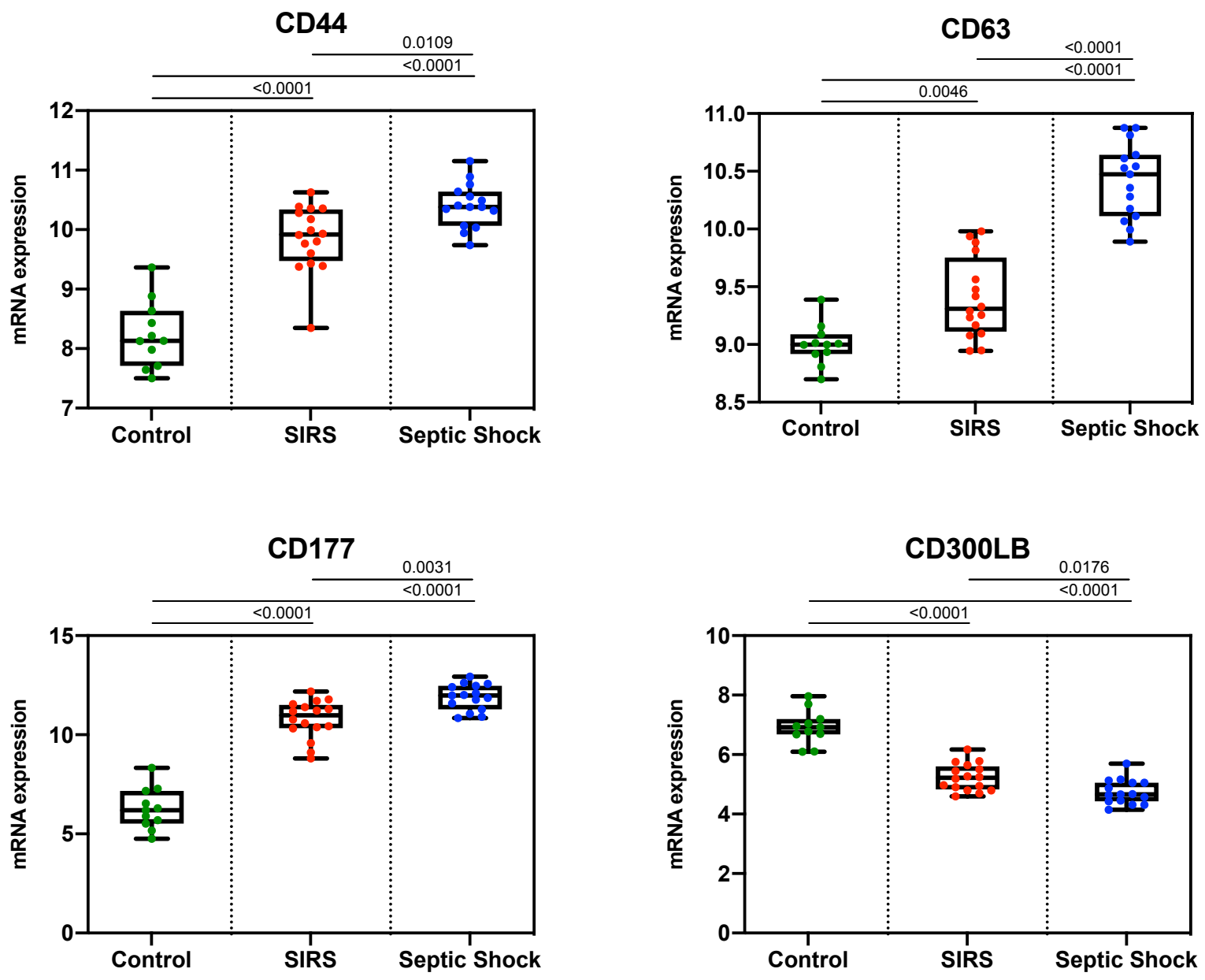

Figure 6: CD44, CD63, CD177 and CD300 LB are differentially expressed in the granulocytes of patients with SIRS and septic shock.

The expression of each gene in the granulocytes of control patients $(n=11)$, patients with SIRS $(n=16)$, or patients with septic shock $(n=15)$ is graphically depicted by box and whisker plot. One-way ANOVA analysis comparing the mean RNA expression value of each gene by patient group (control vs. SIRS, control vs. septic shock, and SIRS vs. septic shock) was performed and the exact $p$-value is indicated on each respective comparison. 


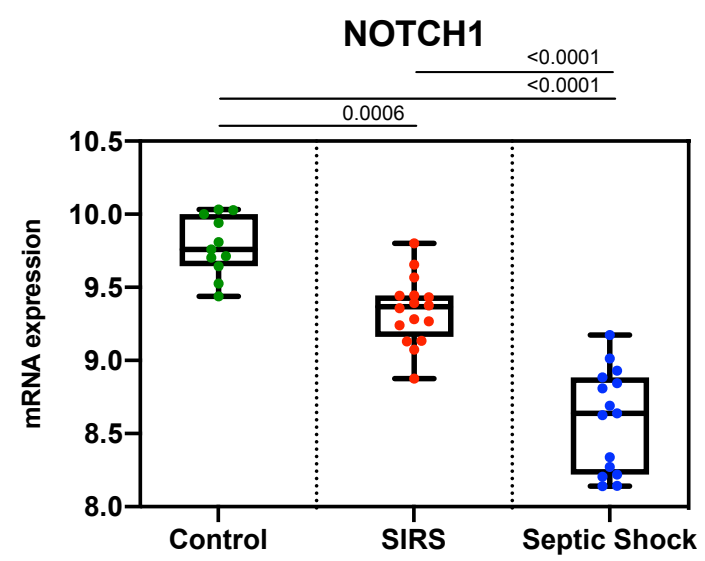

Figure 7: The NOTCH1 signaling molecule is differentially expressed in the granulocytes of patients with SIRS and septic shock.

The expression of each gene in the granulocytes of control patients $(n=11)$, patients with SIRS $(n=16)$, or patients with septic shock $(n=15)$ is graphically depicted by box and whisker plot. One-way ANOVA analysis comparing the mean RNA expression value of each gene by patient group (control vs. SIRS, control vs. septic shock, and SIRS vs. septic shock) was performed and the exact $p$-value is indicated on each respective comparison. 

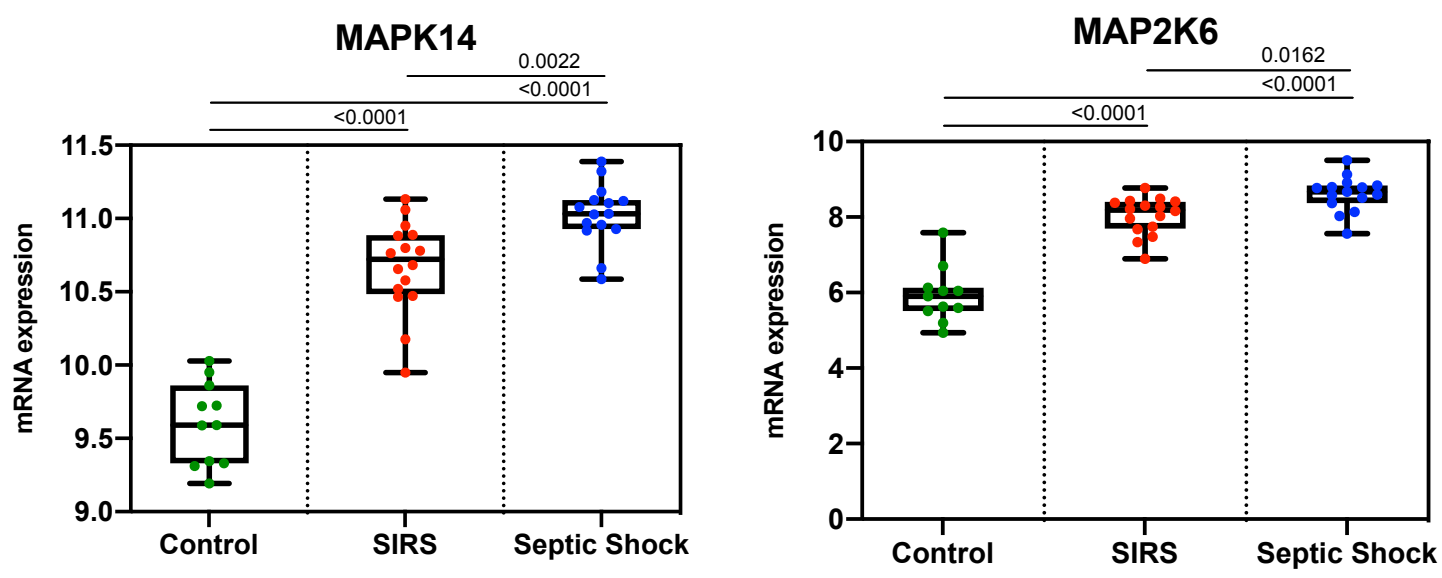

Figure 8: Two map kinase signaling pathway effectors are differentially expressed in the granulocytes of patients with SIRS and septic shock.

The expression of each gene in the granulocytes of control patients $(n=11)$, patients with SIRS $(n=16)$, or patients with septic shock $(n=15)$ is graphically depicted by box and whisker plot. One-way ANOVA analysis comparing the mean RNA expression value of each gene by patient group (control vs. SIRS, control vs. septic shock, and SIRS vs. septic shock) was performed and the exact $\mathrm{p}$-value is indicated on each respective comparison. 

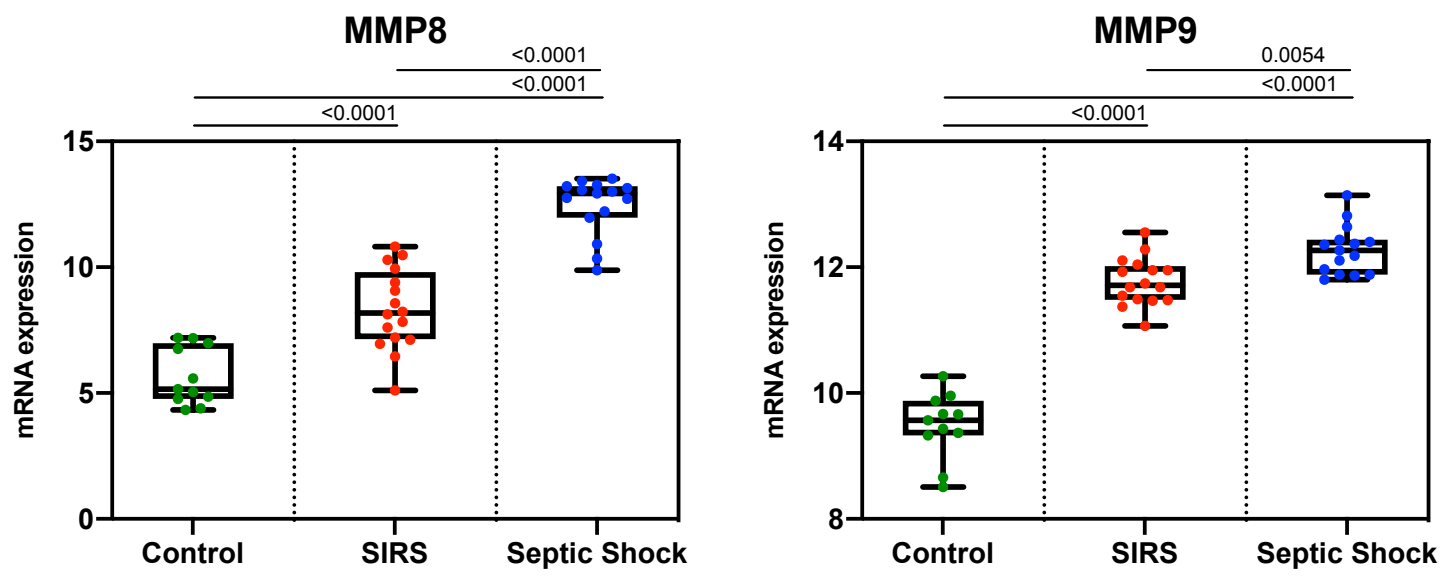

Figure 9: Matrix metalloproteinases MMP8 and MMP9 are differentially expressed in the granulocytes of patients with SIRS and septic shock.

The expression of each gene in the granulocytes of control patients $(n=11)$, patients with SIRS $(n=16)$, or patients with septic shock $(n=15)$ is graphically depicted by box and whisker plot. One-way ANOVA analysis comparing the mean RNA expression value of each gene by patient group (control vs. SIRS, control vs. septic shock, and SIRS vs. septic shock) was performed and the exact $p$-value is indicated on each respective comparison. 

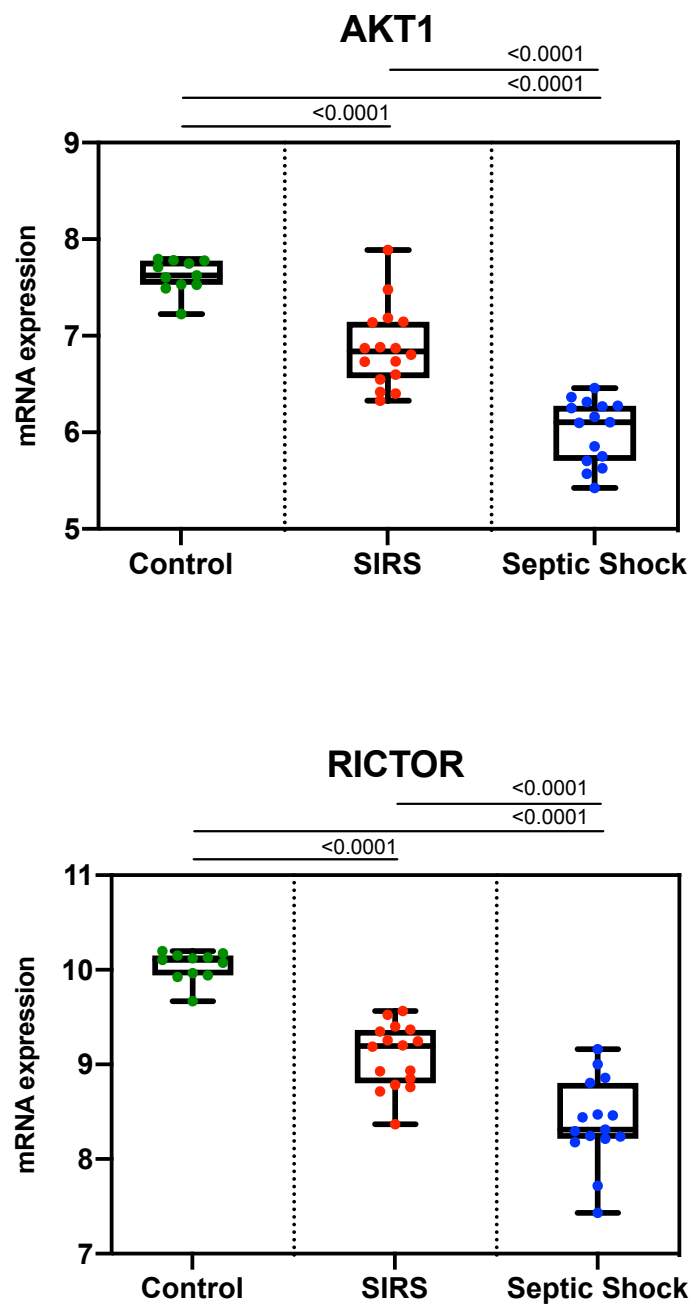

Figure 10: AKT1 and RICTOR are differentially expressed in the granulocytes of patients with SIRS and septic shock.

The expression of each gene in the granulocytes of control patients $(n=11)$, patients with SIRS $(n=16)$, or patients with septic shock $(n=15)$ is graphically depicted by box and whisker plot. One-way ANOVA analysis comparing the mean RNA expression value of each gene by patient group (control vs. SIRS, control vs. septic shock, and SIRS vs. septic shock) was performed and the exact $p$-value is indicated on each respective comparison. 

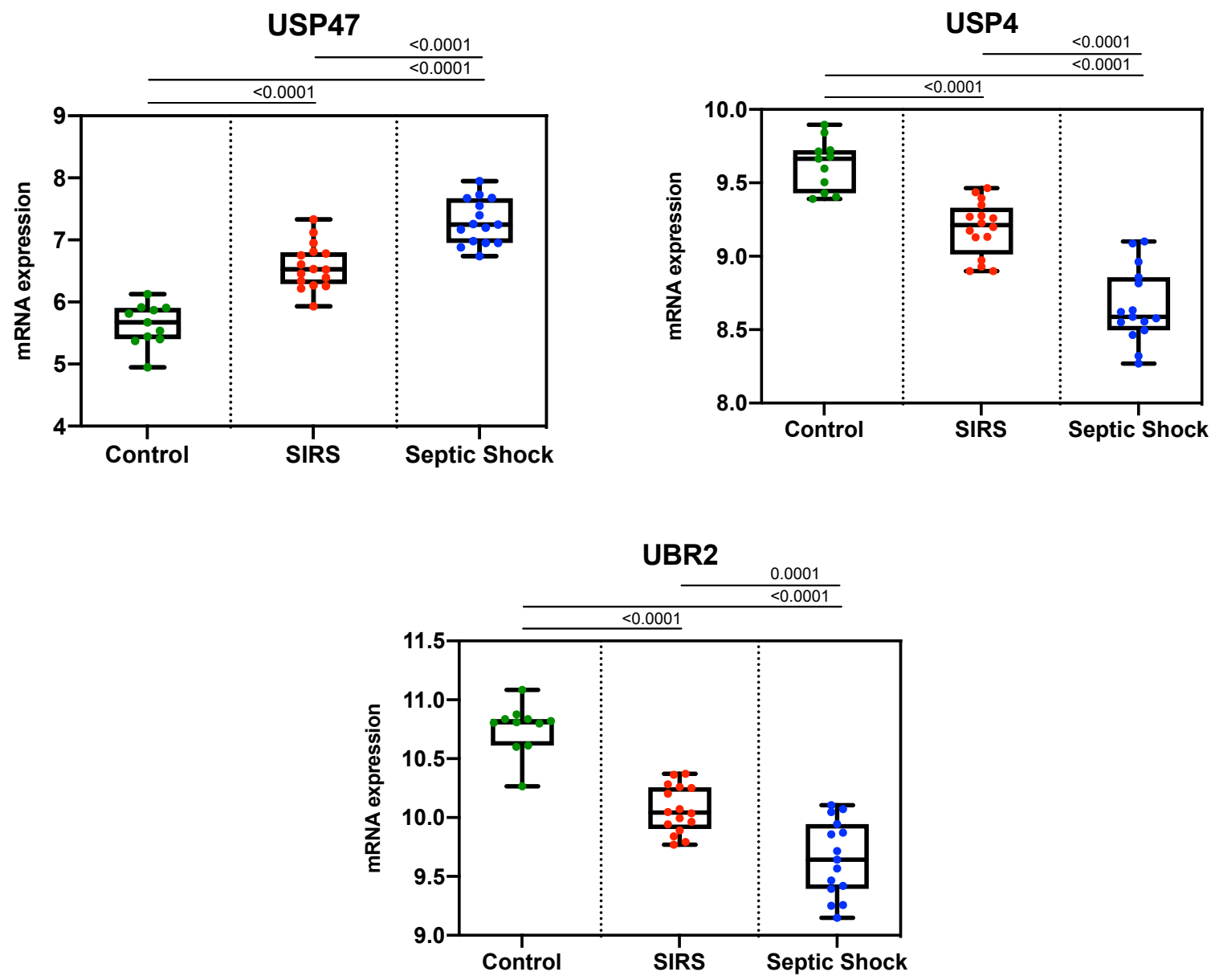

Figure 11: Two deubiquitinases and an E3 ubiquitin ligase are differentially expressed in the granulocytes of patients with SIRS and septic shock.

The expression of each gene in the granulocytes of control patients $(n=11)$, patients with SIRS $(n=16)$, or patients with septic shock $(n=15)$ is graphically depicted by box and whisker plot. One-way ANOVA analysis comparing the mean RNA expression value of each gene by patient group (control vs. SIRS, control vs. septic shock, and SIRS vs. septic shock) was performed and the exact $p$-value is indicated on each respective comparison. 

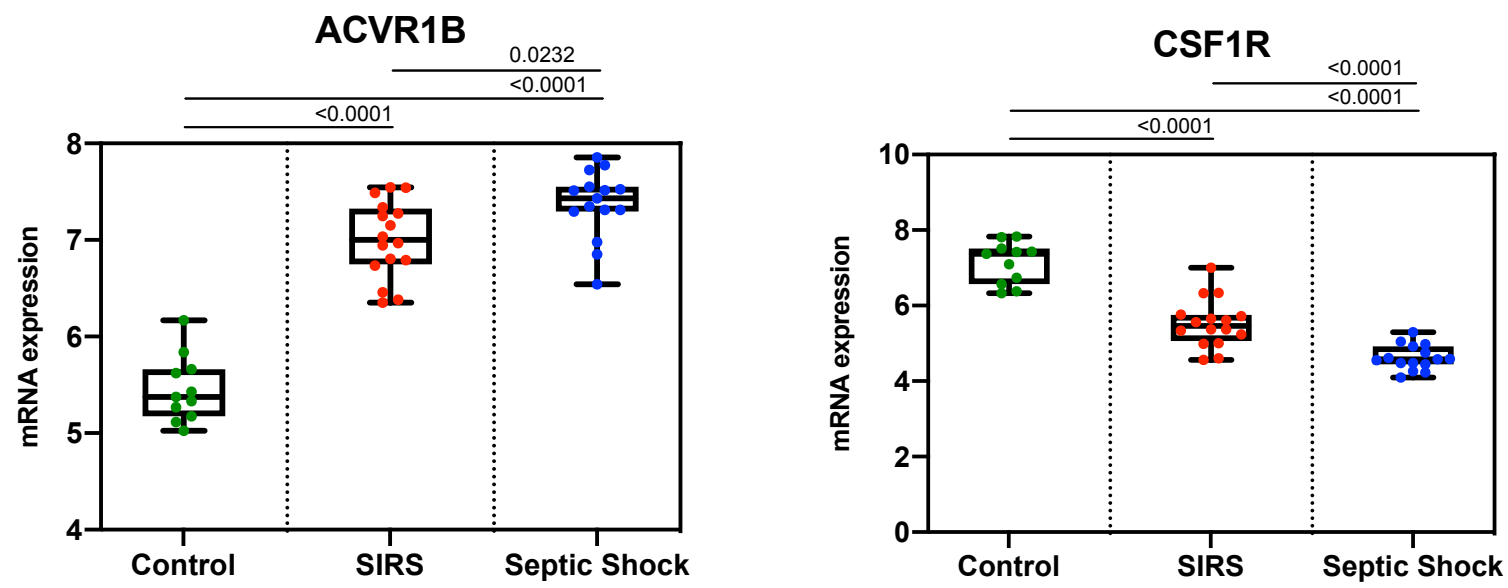

Figure 12: Cell surface receptors ACVR1B and CSF1R are differentially expressed in the granulocytes of patients with SIRS and septic shock.

The expression of each gene in the granulocytes of control patients $(n=11)$, patients with SIRS $(n=16)$, or patients with septic shock $(n=15)$ is graphically depicted by box and whisker plot. One-way ANOVA analysis comparing the mean RNA expression value of each gene by patient group (control vs. SIRS, control vs. septic shock, and SIRS vs. septic shock) was performed and the exact $p$-value is indicated on each respective comparison. 

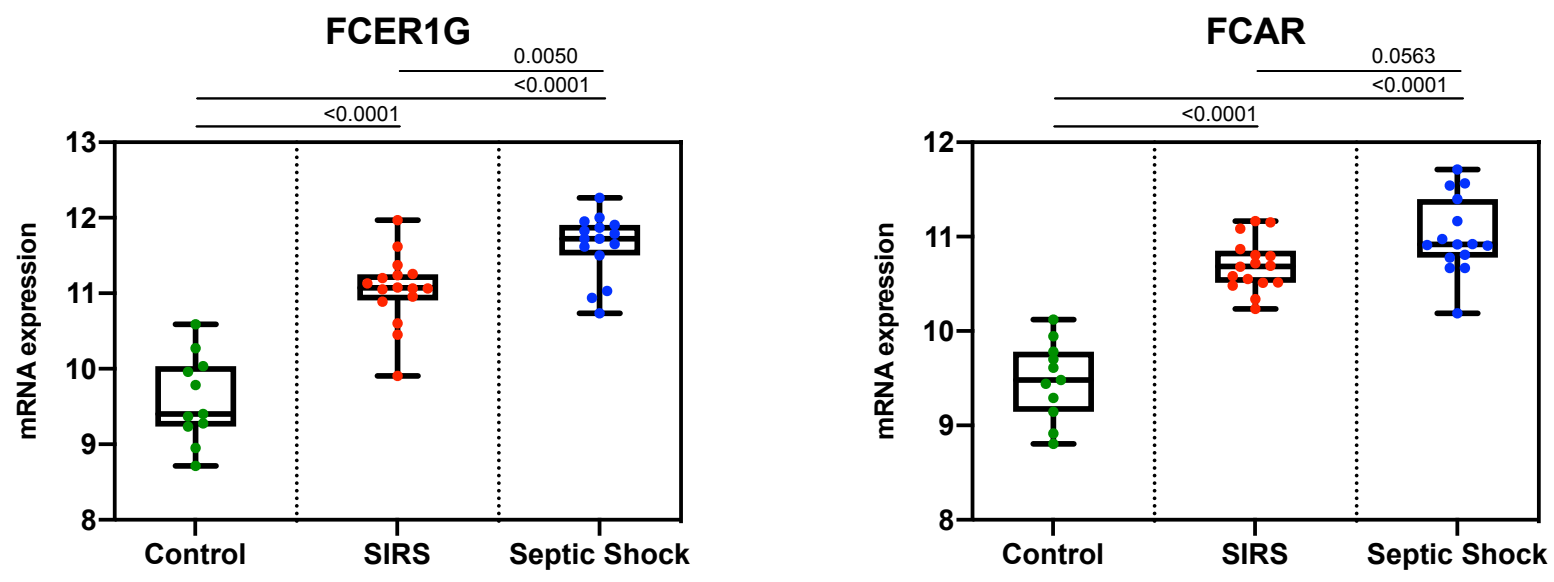

Figure 13: Genes encoding Fc receptor subunits for IgE and IgA are differentially expressed in the granulocytes of patients with SIRS and septic shock.

The expression of each gene in the granulocytes of control patients $(n=11)$, patients with SIRS $(n=16)$, or patients with septic shock $(n=15)$ is graphically depicted by box and whisker plot. One-way ANOVA analysis comparing the mean RNA expression value of each gene by patient group (control vs. SIRS, control vs. septic shock, and SIRS vs. septic shock) was performed and the exact $p$-value is indicated on each respective comparison. 

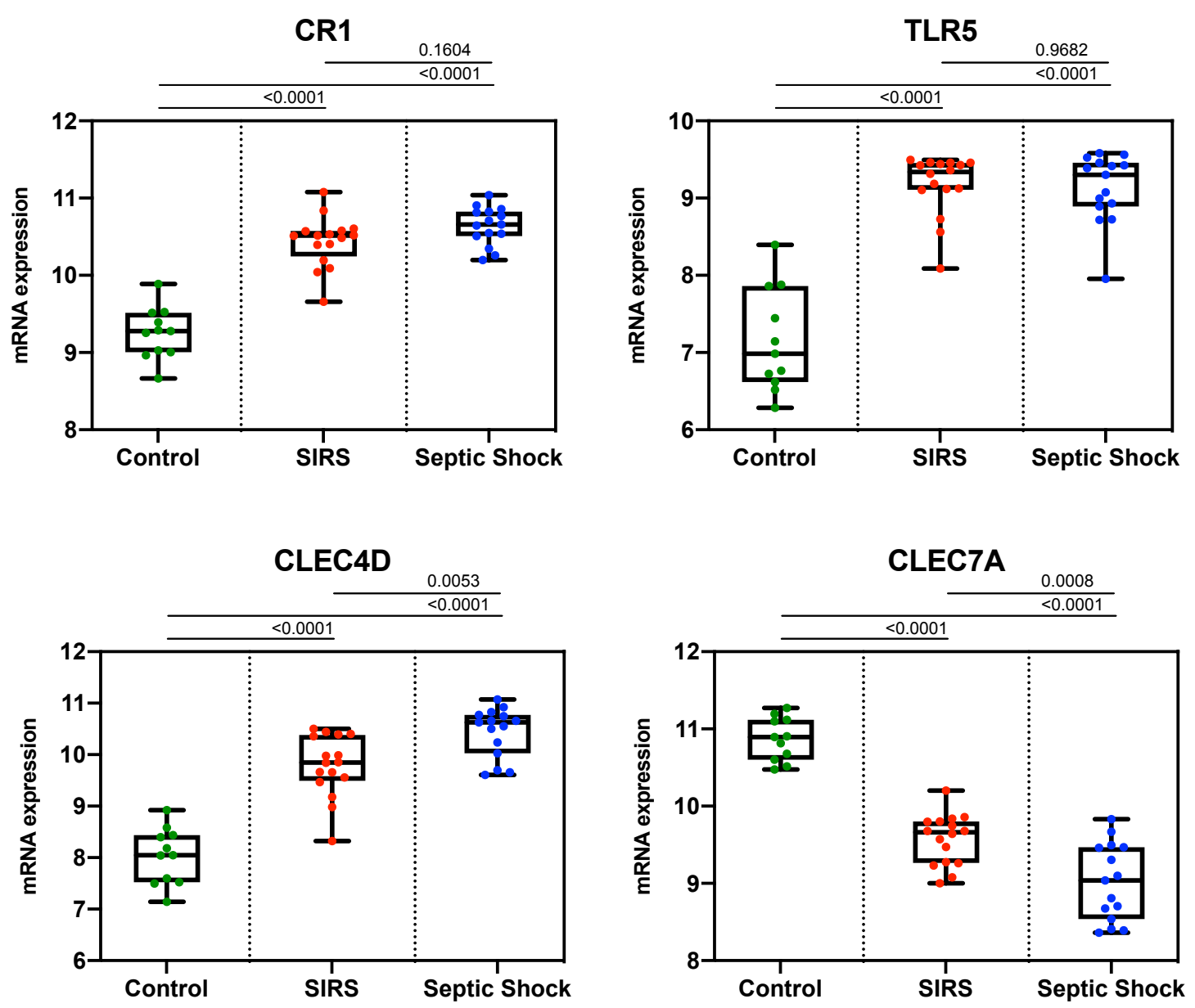

Figure 14: Four cell surface receptors involved in innate immune signaling are differentially expressed in the granulocytes of patients with SIRS and septic shock.

The expression of each gene in the granulocytes of control patients $(n=11)$, patients with SIRS $(n=16)$, or patients with septic shock $(n=15)$ is graphically depicted by box and whisker plot. One-way ANOVA analysis comparing the mean RNA expression value of each gene by patient group (control vs. SIRS, control vs. septic shock, and SIRS vs. septic shock) was performed and the exact $p$-value is indicated on each respective comparison. 

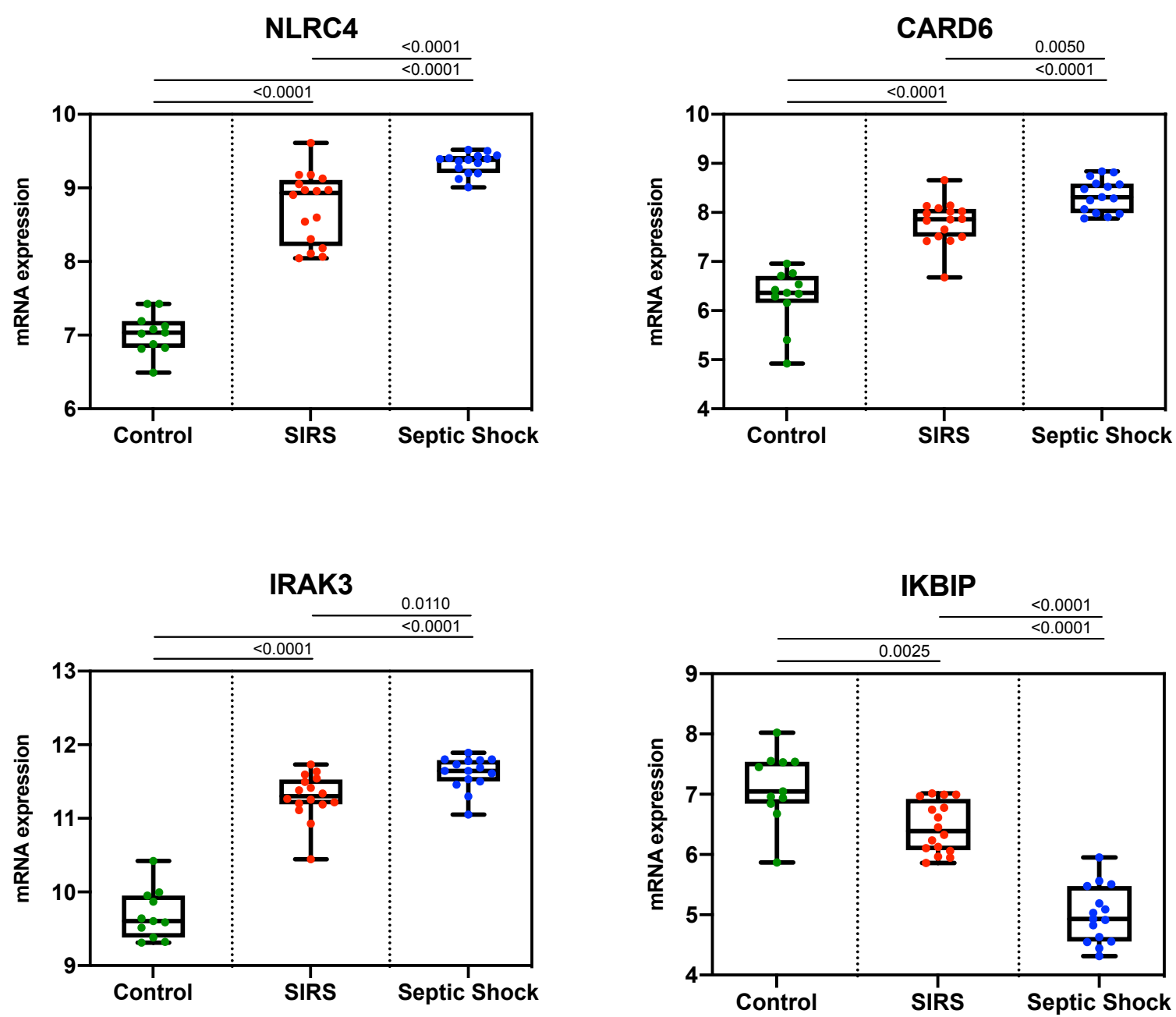

Figure 15: Four intracellular effectors of innate immune signaling are differentially expressed in the granulocytes of patients with SIRS and septic shock.

The expression of each gene in the granulocytes of control patients $(n=11)$, patients with SIRS $(n=16)$, or patients with septic shock $(n=15)$ is graphically depicted by box and whisker plot. One-way ANOVA analysis comparing the mean RNA expression value of each gene by patient group (control vs. SIRS, control vs. septic shock, and SIRS vs. septic shock) was performed and the exact $p$-value is indicated on each respective comparison. 

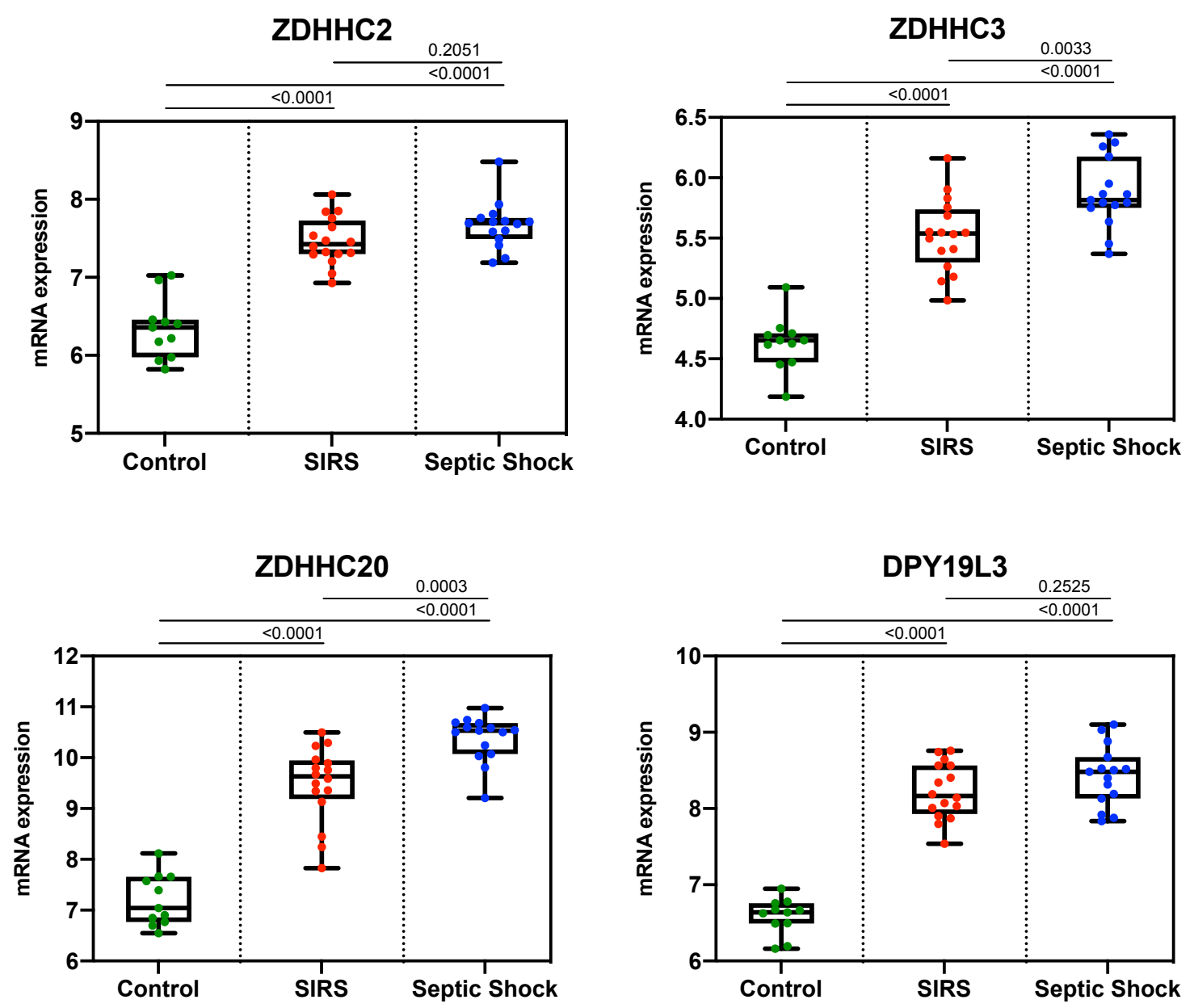

Figure 16: Three palmitoyltransferases and a mannosyltransferase are differentially expressed in the granulocytes of patients with SIRS and septic shock.

The expression of each gene in the granulocytes of control patients $(n=11)$, patients with SIRS $(n=16)$, or patients with septic shock $(n=15)$ is graphically depicted by box and whisker plot. One-way ANOVA analysis comparing the mean RNA expression value of each gene by patient group (control vs. SIRS, control vs. septic shock, and SIRS vs. septic shock) was performed and the exact $p$-value is indicated on each respective comparison. 

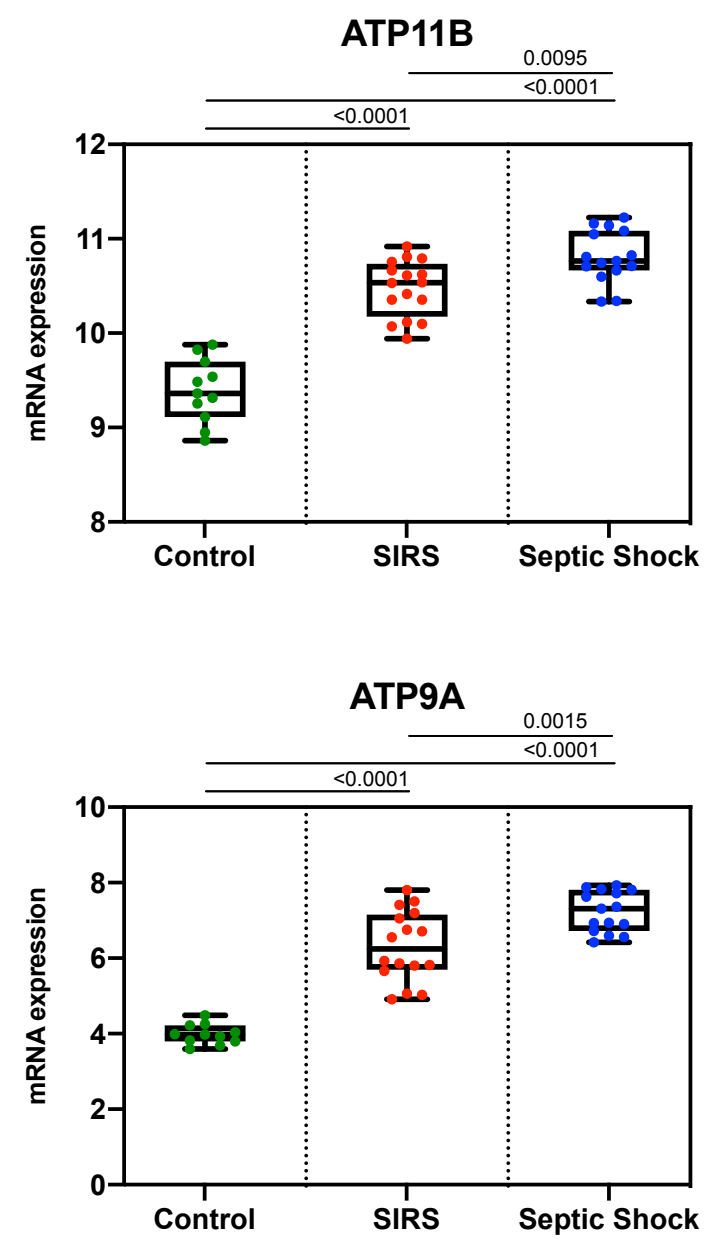

Figure 17: An ATP flippase and an ATPase are differentially expressed in the granulocytes of patients with SIRS and septic shock.

The expression of each gene in the granulocytes of control patients $(n=11)$, patients with SIRS $(n=16)$, or patients with septic shock $(n=15)$ is graphically depicted by box and whisker plot. One-way ANOVA analysis comparing the mean RNA expression value of each gene by patient group (control vs. SIRS, control vs. septic shock, and SIRS vs. septic shock) was performed and the exact $p$-value is indicated on each respective comparison. 

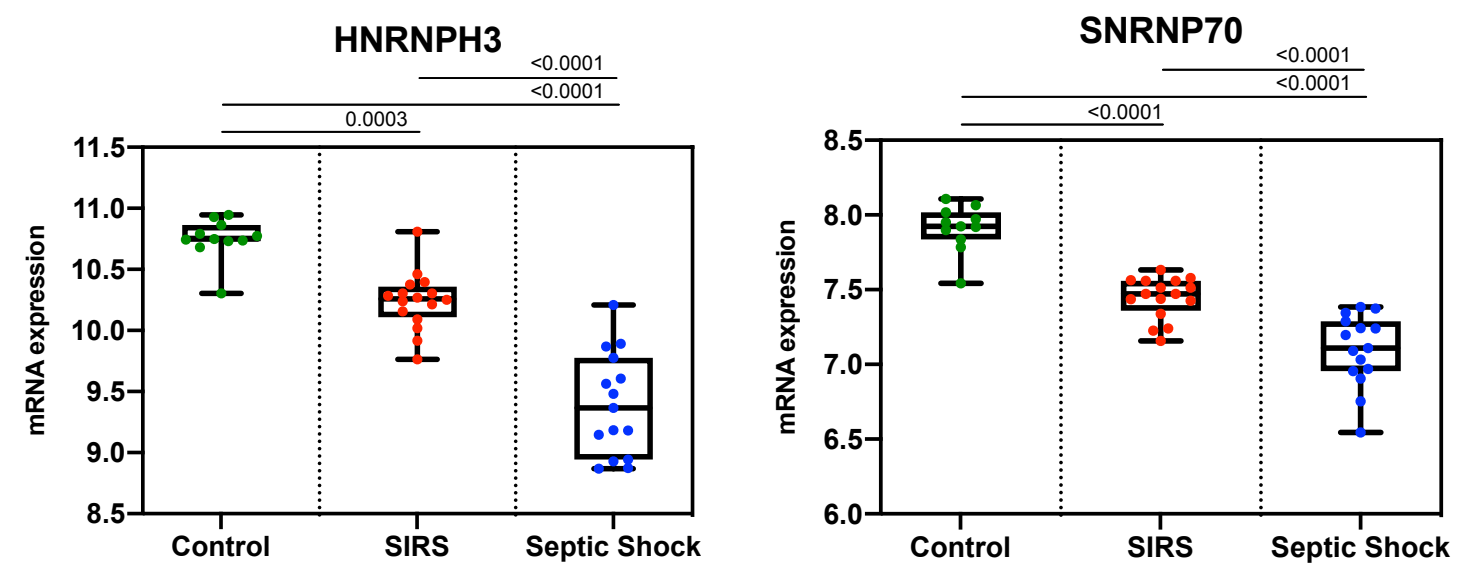

Figure 18: Genes encoding ribonucleoproteins, or RNPs are differentially expressed in the granulocytes of patients with SIRS and septic shock.

The expression of each gene in the granulocytes of control patients $(n=11)$, patients with SIRS $(n=16)$, or patients with septic shock $(n=15)$ is graphically depicted by box and whisker plot. One-way ANOVA analysis comparing the mean RNA expression value of each gene by patient group (control vs. SIRS, control vs. septic shock, and SIRS vs. septic shock) was performed and the exact $p$-value is indicated on each respective comparison. 

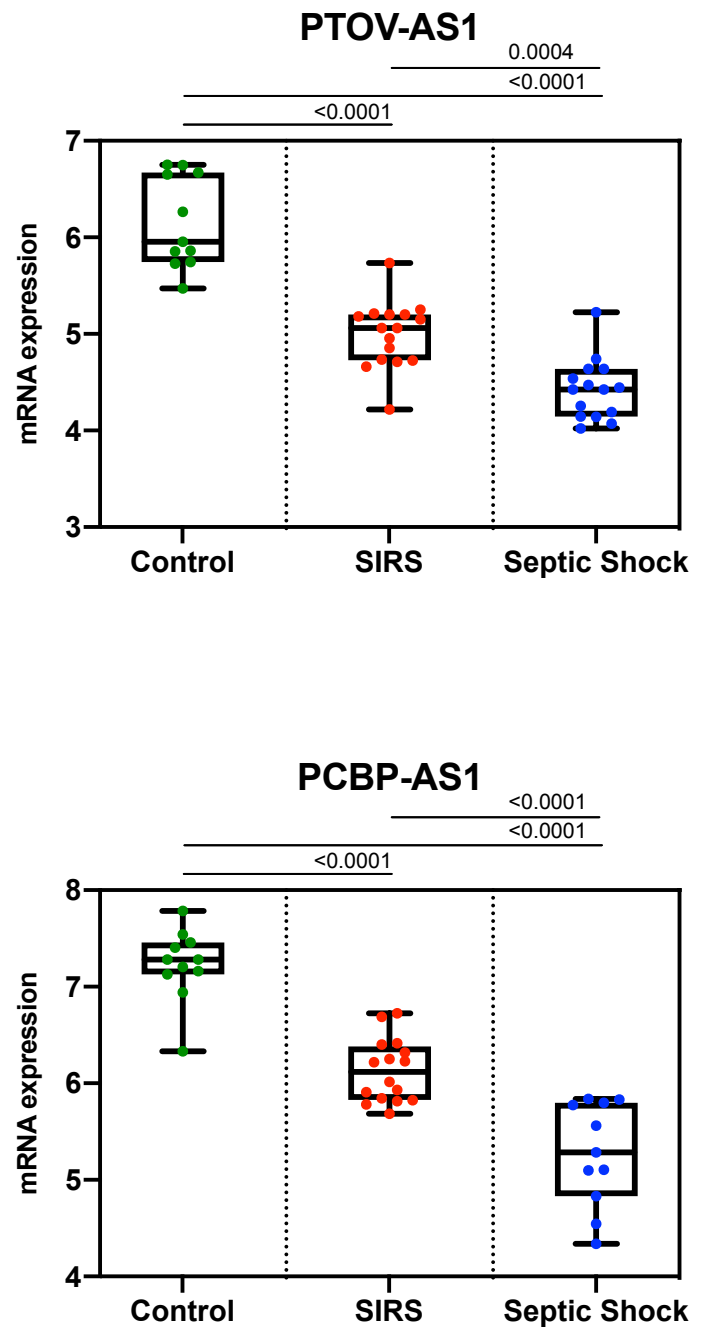

Figure 19: Two non-coding RNA transcripts were differentially expressed in the granulocytes of patients with SIRS and septic shock.

The expression of each gene in the granulocytes of control patients $(n=11)$, patients with SIRS $(n=16)$, or patients with septic shock $(n=15)$ is graphically depicted by box and whisker plot. One-way ANOVA analysis comparing the mean RNA expression value of each gene by patient group (control vs. SIRS, control vs. septic shock, and SIRS vs. septic shock) was performed and the exact $p$-value is indicated on each respective comparison. 

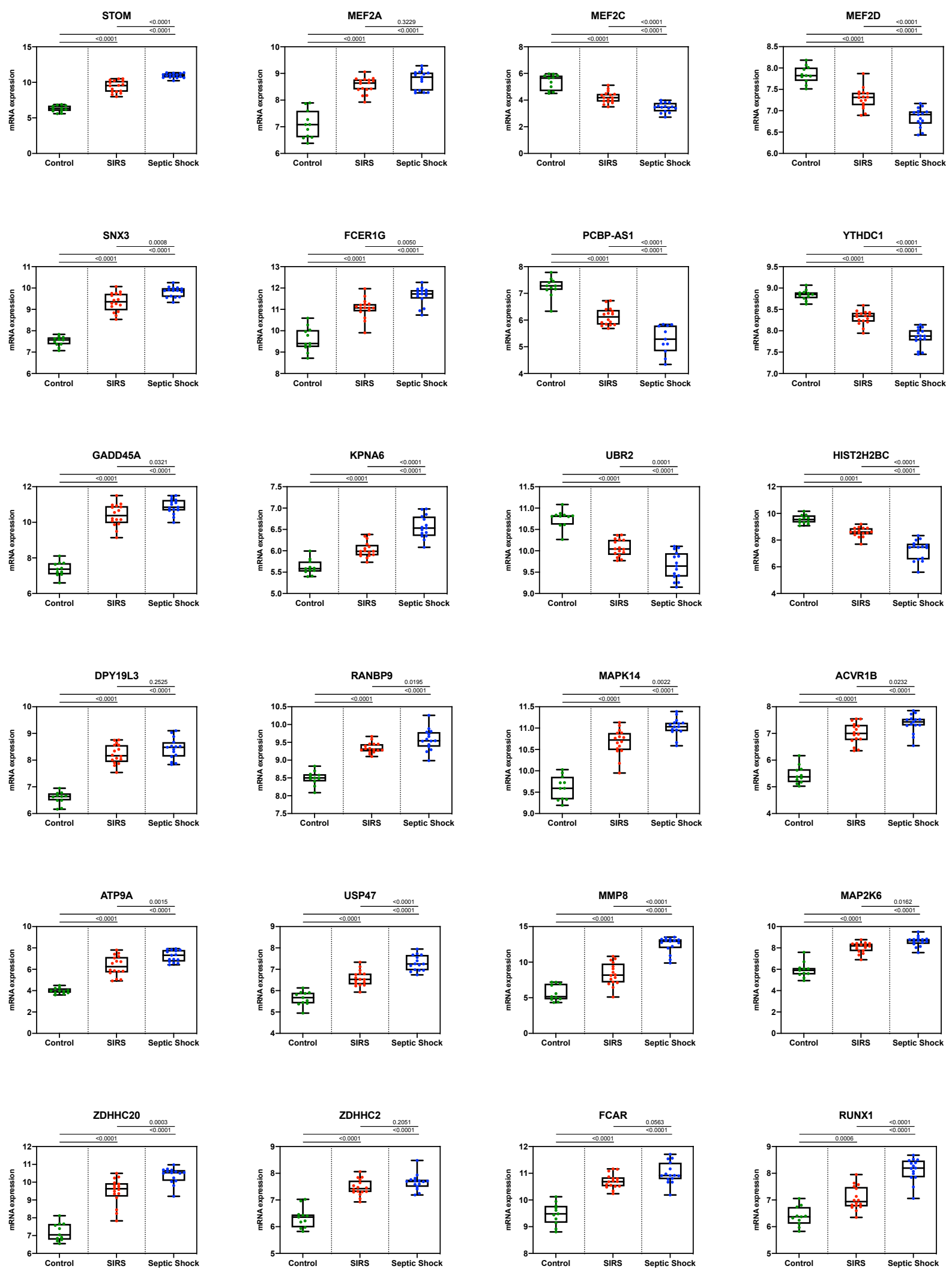

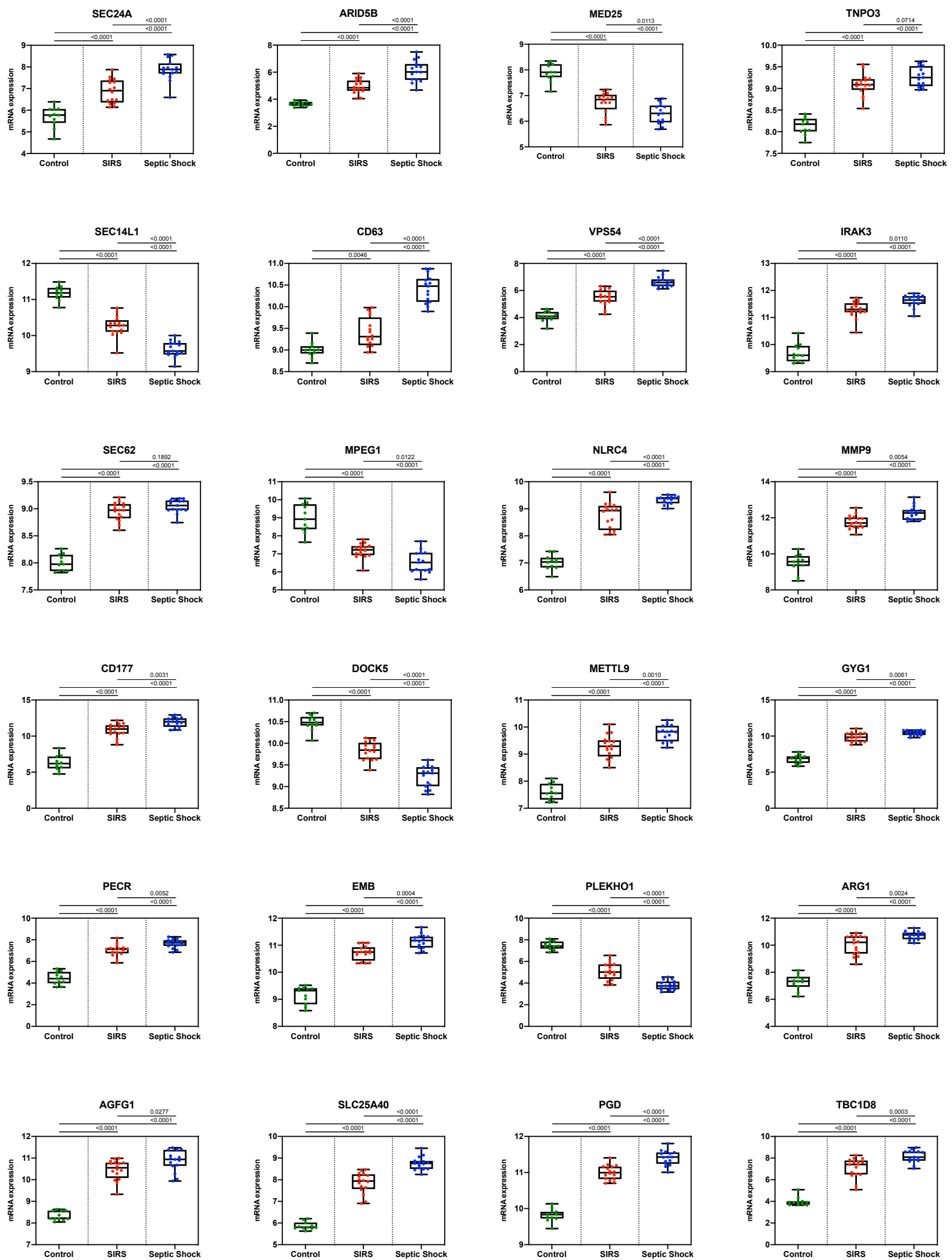

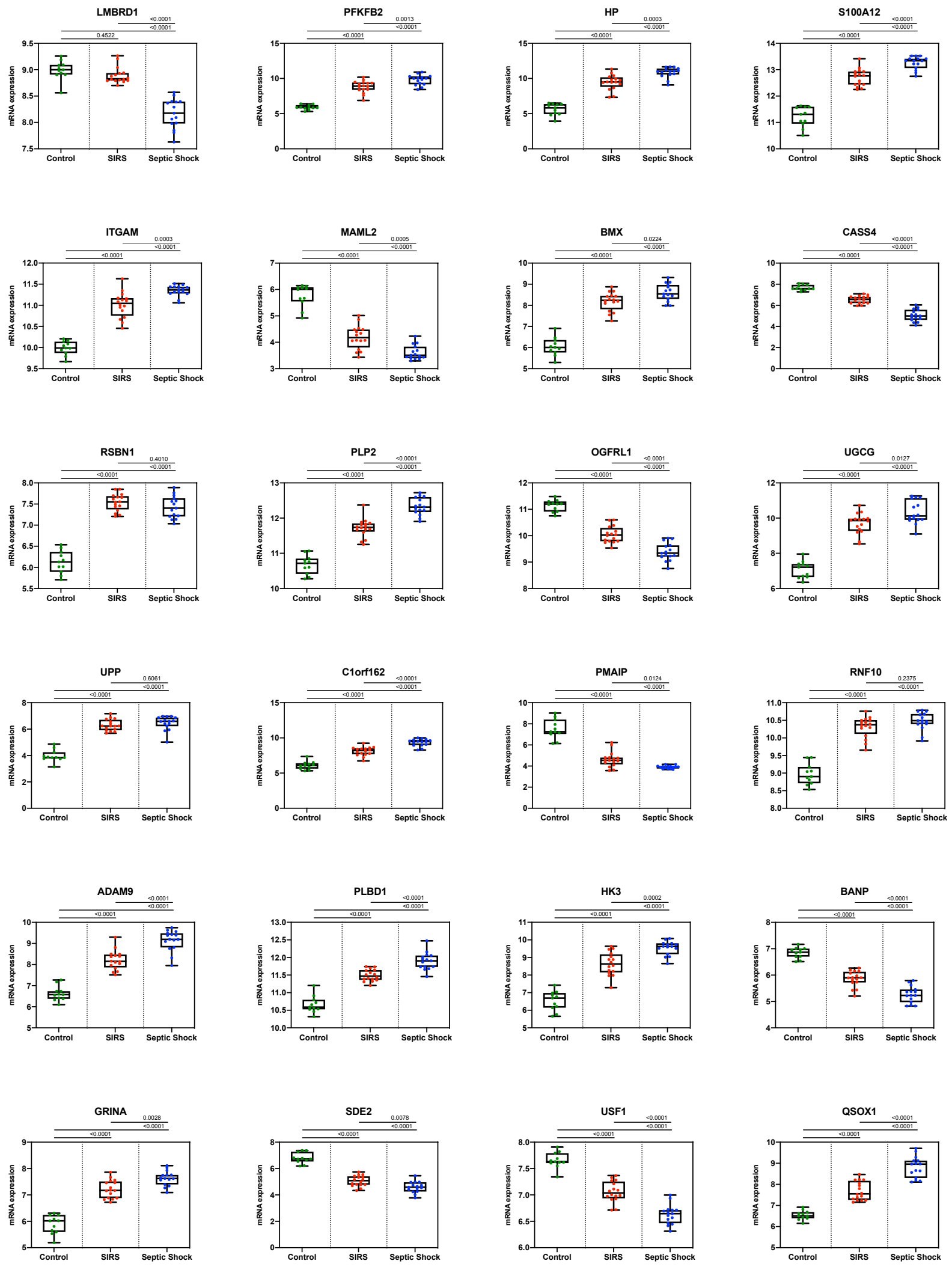

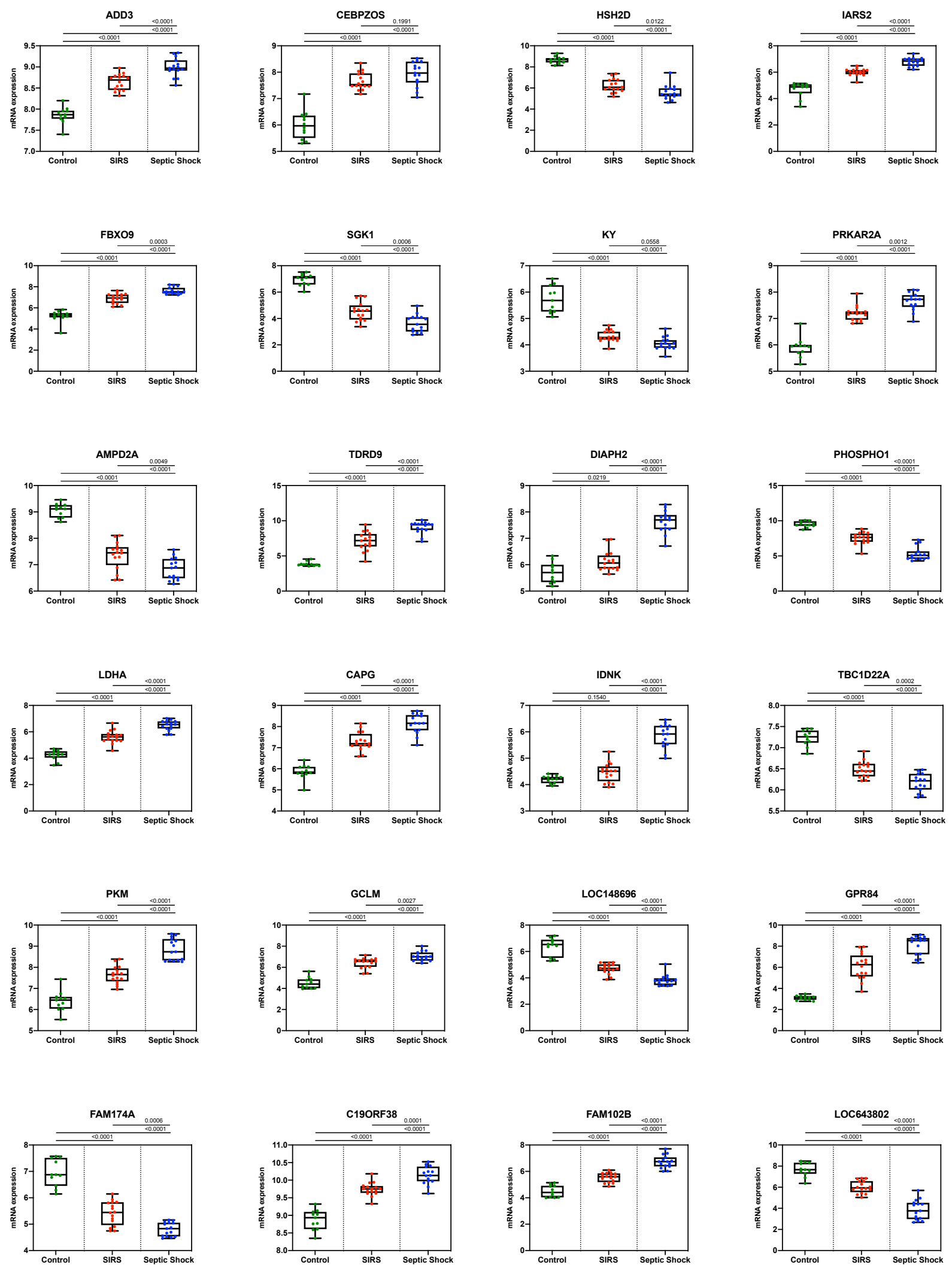

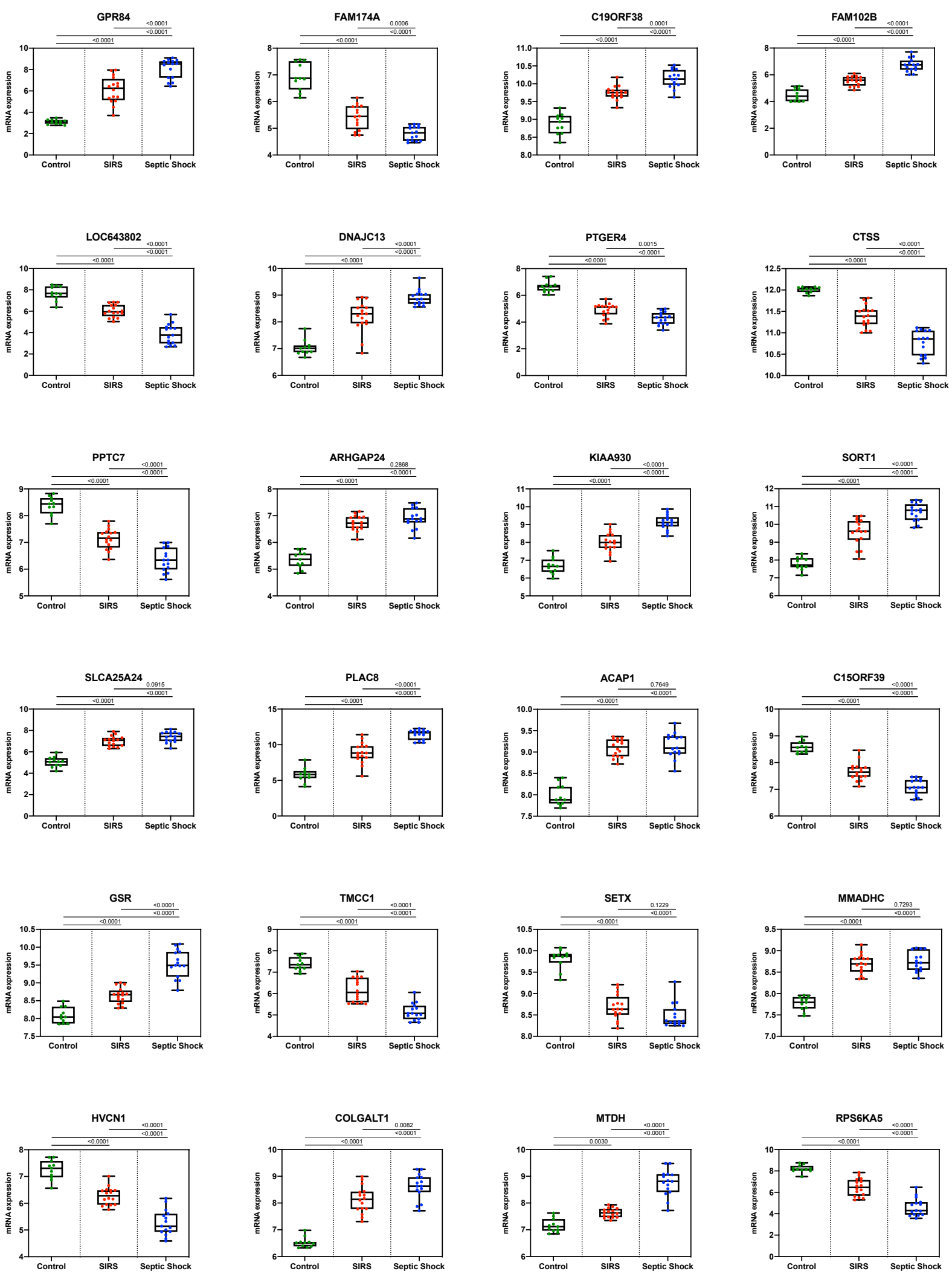

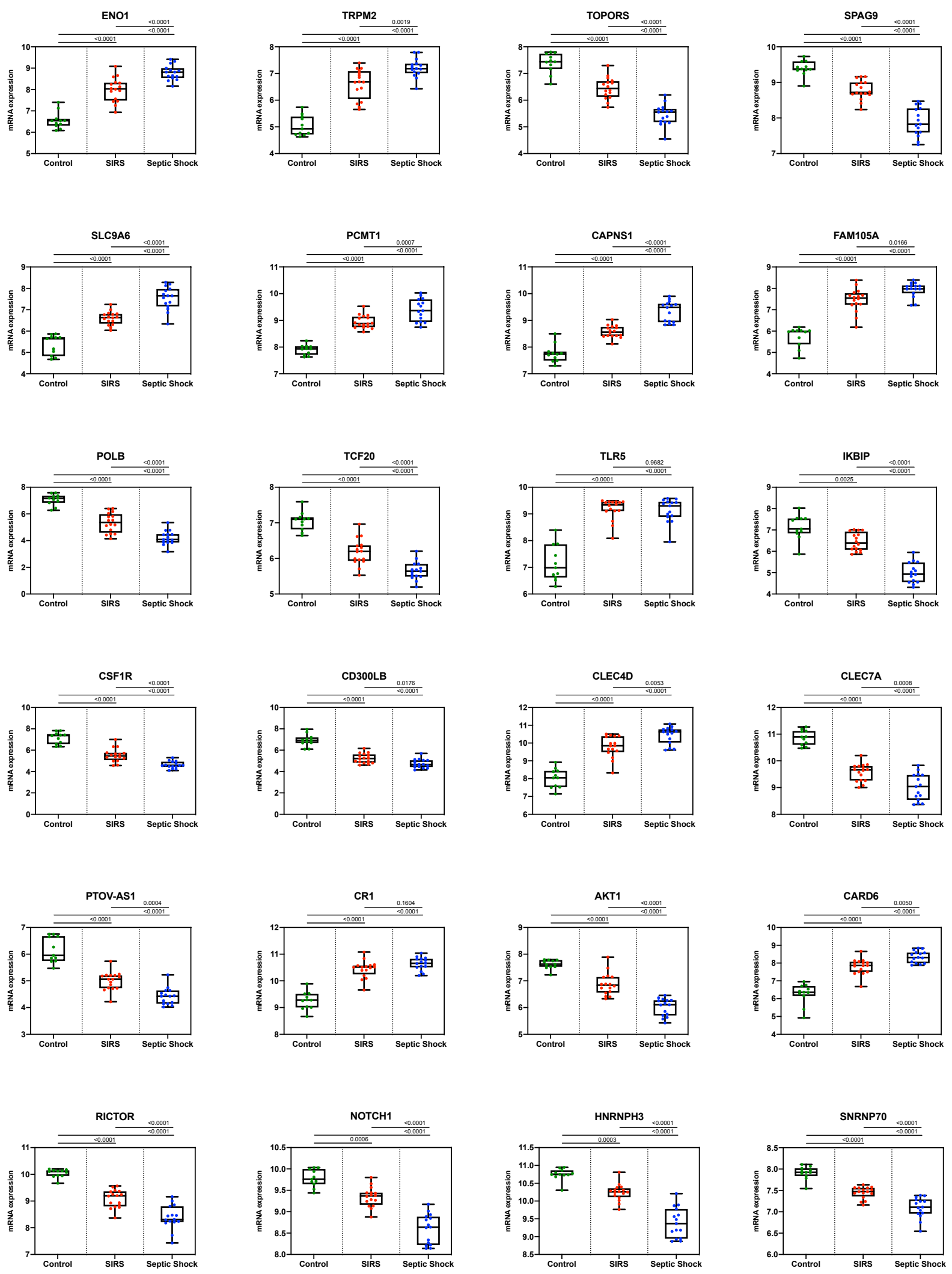

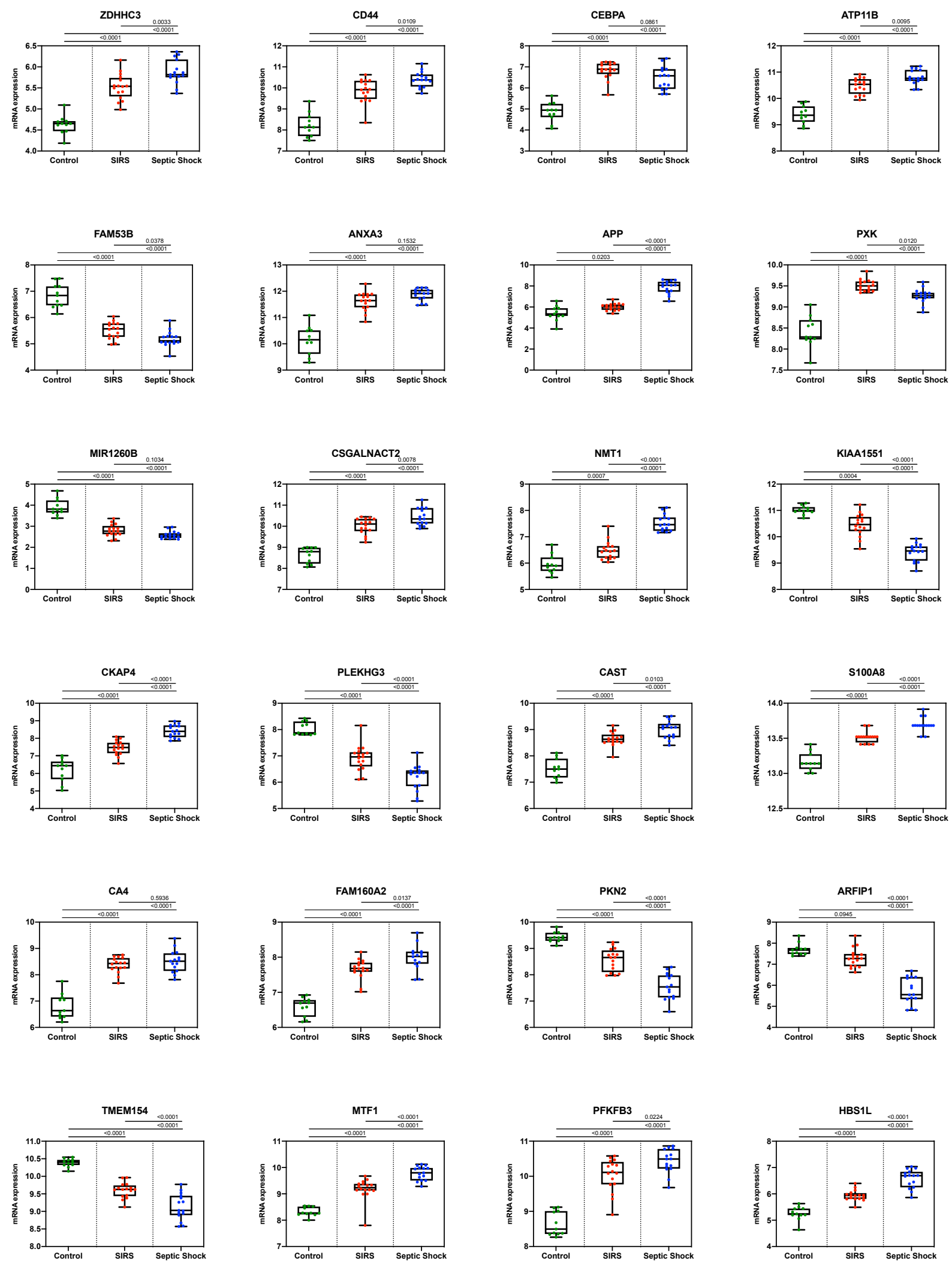

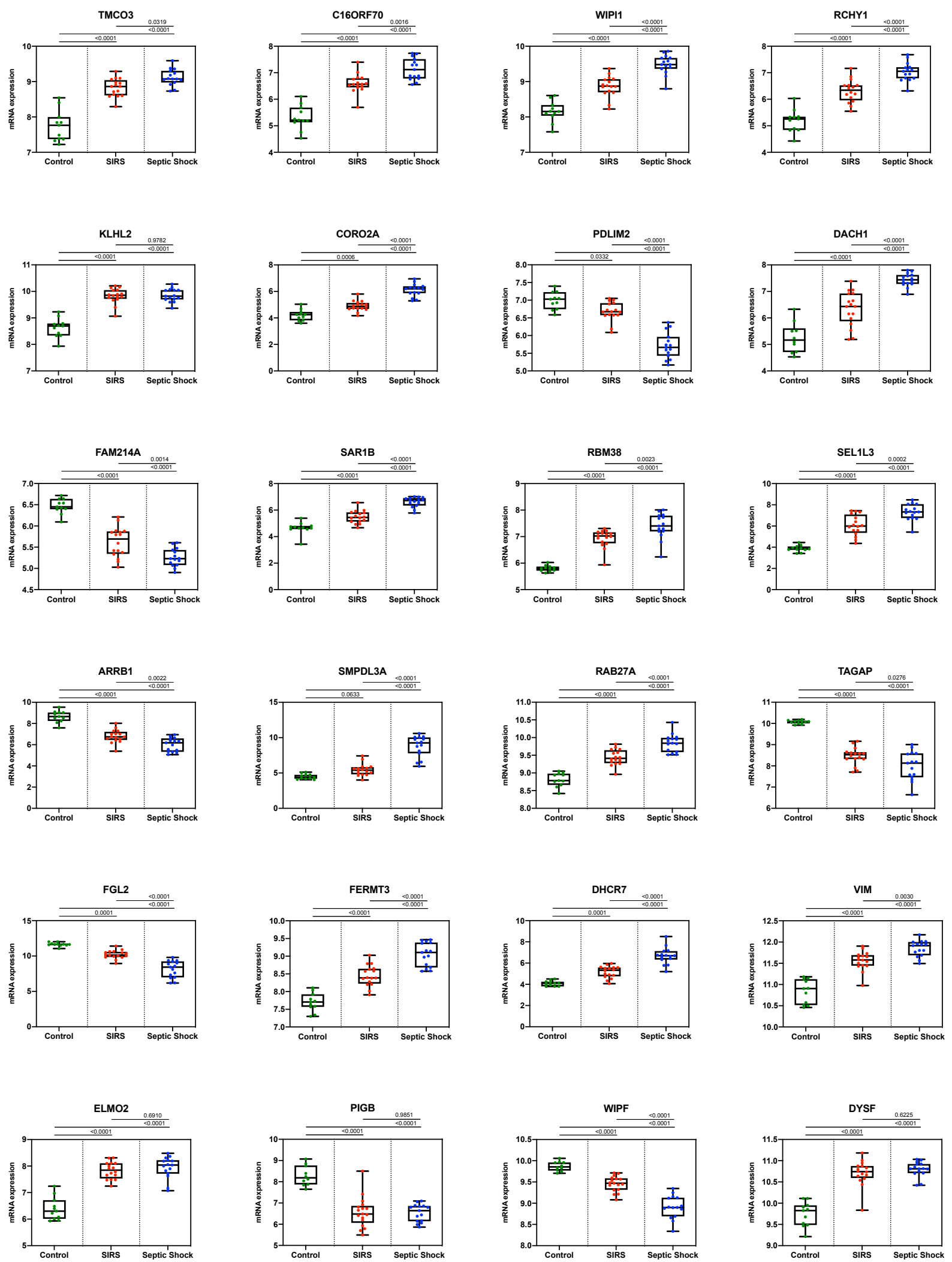

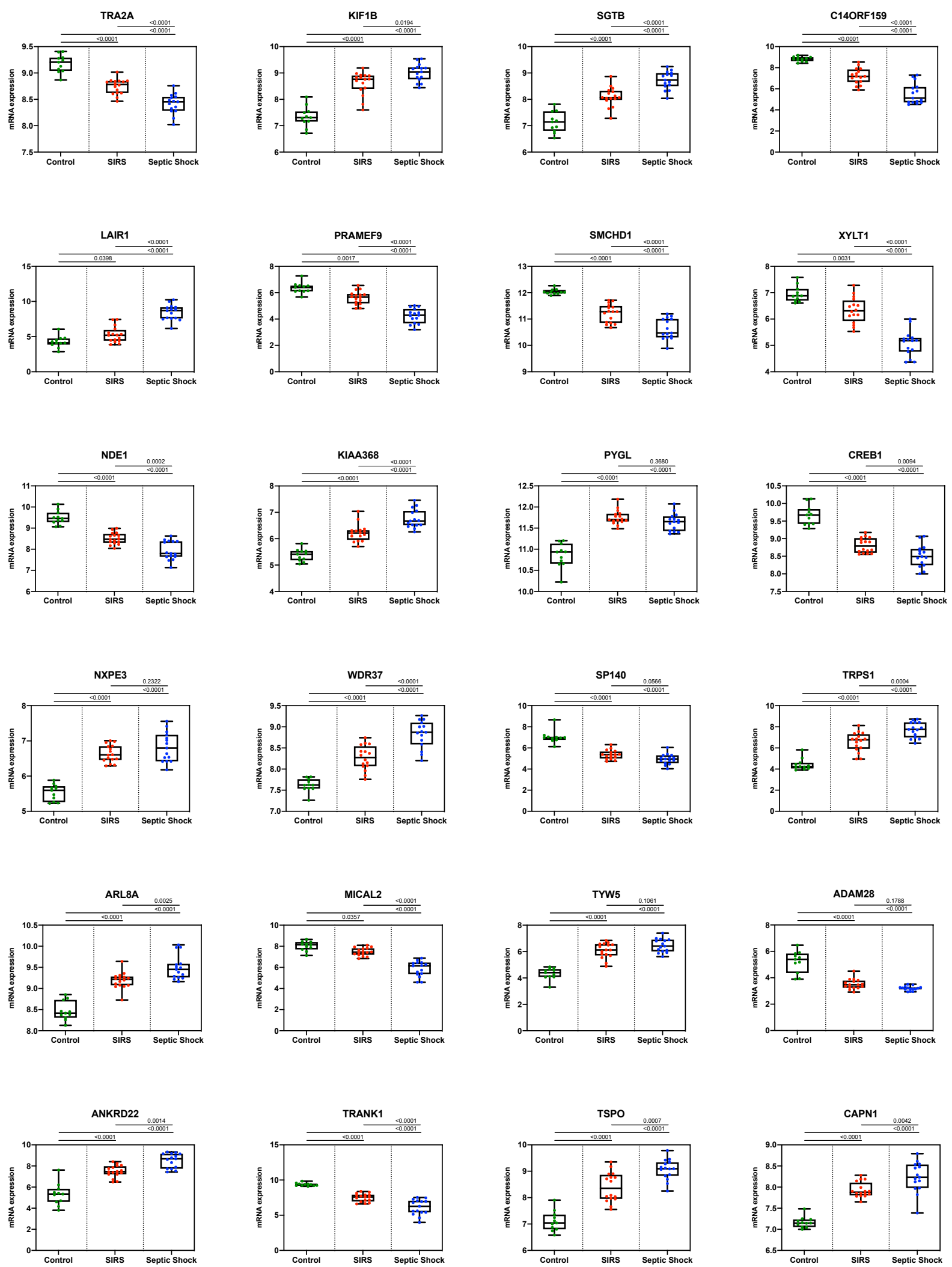

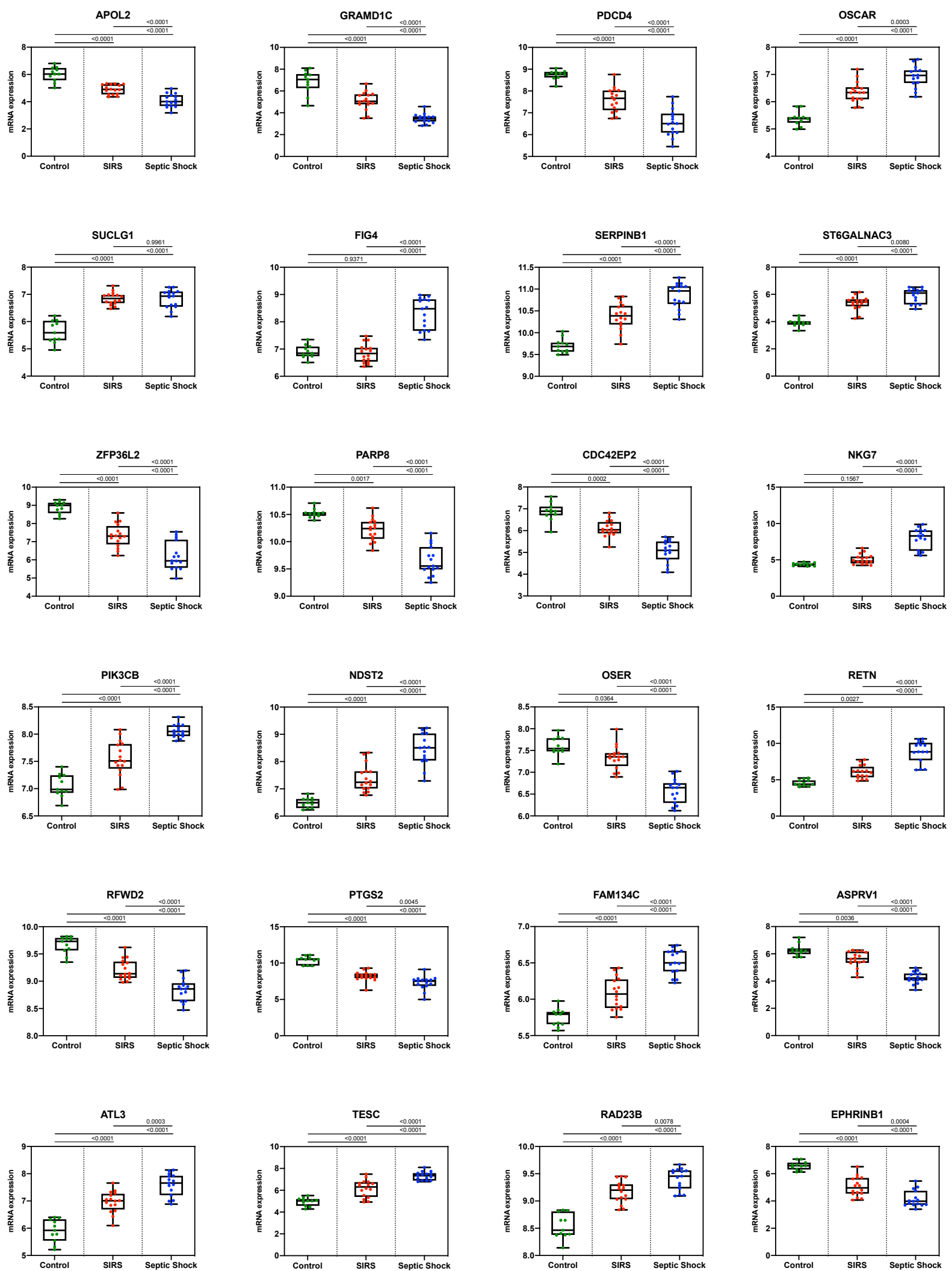

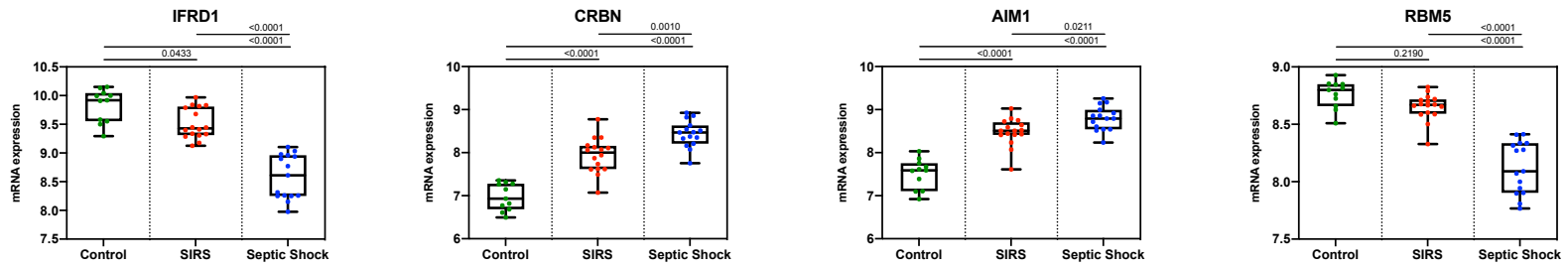

Figure 20: SIRS represents an intermediate gene expression state between granulocytes of patients with septic shock and the granulocytes of control patients.

The expression of each gene in the granulocytes of control patients $(n=11)$, patients with SIRS $(n=16)$, or patients with septic shock $(n=15)$ is graphically depicted by box and whisker plot. One-way ANOVA analysis comparing the mean RNA expression value of each gene by patient group (control vs. SIRS, control vs. septic shock, and SIRS vs. septic shock) was performed and the exact $p$-value is indicated on each respective comparison. 